\title{
Covariance estimation via sparse Kronecker structures
}

\author{
CHENLEI LENG ${ }^{1}$ and GUANGMING PAN ${ }^{2}$ \\ ${ }^{1}$ Department of Statistics, University of Warwick, CoventryCV4 7AL, UK. E-mail: C.Leng@warwick.ac.uk \\ ${ }^{2}$ School of Physical and Mathematical Sciences, Nanyang Technological University, Singapore 637371, \\ Republic of Singapore. E-mail: gmpan@ntu.edu.sg
}

The problem of estimating covariance matrices is central to statistical analysis and is extensively addressed when data are vectors. This paper studies a novel Kronecker-structured approach for estimating such matrices when data are matrices and arrays. Focusing on matrix-variate data, we present simple approaches to estimate the row and the column correlation matrices, formulated separately via convex optimization. We also discuss simple thresholding estimators motivated by the recent development in the literature. Nonasymptotic results show that the proposed method greatly outperforms methods that ignore the matrix structure of the data. In particular, our framework allows the dimensionality of data to be arbitrary order even for fixed sample size, and works for flexible distributions beyond normality. Simulations and data analysis further confirm the competitiveness of the method. An extension to general array-data is also outlined.

Keywords: covariance matrix; Kronecker structure; matrix data; non-asymptotic bound

\section{Introduction}

Matrix and array observations are becoming increasingly available in the big data era thanks to the rapid advance in the information technology and the need to store data in structured forms; see, for example, Li, Kim and Altman [14], Hoff [11], Leng and Tang [13], Zhou, Li and Zhu [24], and Zhou et al. [25]. Consider independent and identically distributed matrixvariates $X_{1}, \ldots, X_{n} \in \mathbb{R}^{p \times q}$ that are realizations of a matrix random variable $X$ following a matrix-variate distribution (Gupta and Nagar [10]). Writing vec as the vector operator that stacks the columns of a matrix into a vector, we denote

$$
\operatorname{var}\left(\operatorname{vec}\left(X_{k}\right)\right)=\Gamma
$$

as the $p q \times p q$ dimensional covariance matrix. Without loss of generality, we assume that $\mathrm{E}\left(X_{k}\right)$ is known or a consistent estimator of $\mathrm{E}\left(X_{k}\right)$ such as the sample mean is available. For the latter case, we require $n>1$. In the sequel, we work with $X_{k}-\mathrm{E}\left(X_{k}\right)$.

The covariance matrix $\Gamma$ plays an indispensable role in multivariate data analysis and is a central quantity for estimation and inference. To begin with, a simple estimate of $\Gamma$ is the familiar sample covariance matrix after these observations are vectorized. However, whenever the data dimension $p q$ is larger than the sample size $n$, this estimator can be of little use due to its singularity. Based on this observation, a plethora of approaches, built upon various sparsity assumptions on $\Gamma$, have attracted increasing attention. See, for example, Bickel and Levina [3,4], 
Rothman, Levina and Zhu [17], Cai and Liu [6], Bien and Tibshirani [5], Rothman [15], Xue, Ma and Zou [21], and Cui, Leng and Sun [8].

Stacking matrices into vectors incurs a loss of information in the matrix form of the data. An attractive alternative is to assume (Hoff [11], Leng and Tang [13], Tsiligkaridis and Hero [19])

$$
\Gamma=\Psi \otimes \Sigma,
$$

where, loosely speaking, $\Psi=\left(\psi_{i j}\right) \in \mathbb{R}^{q \times q}$ depicts the covariance of the columns of $X_{i}$ and $\Sigma=\left(\sigma_{i j}\right) \in \mathbb{R}^{p \times p}$ that of the rows. Using a Kronecker product for the overall covariance matrix $\Gamma$ retains the matrix structure of the data. Another immediate advantage is that the number of the unknown parameters in $\Gamma$ reduces from an order of $p^{2} q^{2}$ to an order of $p^{2}+q^{2}$, making the problem more tractable. As will become clear, with appropriate sparsity assumptions on $\Psi$ and $\Sigma$, this decomposition enables one to estimate $\Gamma$ at a higher rate of convergence, and allows substantially larger dimensional covariances to be estimated, even with a fixed sample size. Without considering sparsity, Srivastava, von Rosen and von Rosen [18] estimated the Kronecker structure when $p$ and $q$ are fixed. There are also a growing number of papers on estimating the concentration matrix $\Gamma^{-1}$ via a Kronecker product representation by estimating sparse concentration matrices $\Psi^{-1}$ and $\Sigma^{-1}$ (Allen and Tibshirani [1], Yin and Li [22], Leng and Tang [13], Zhou [26]). These papers assume matrix normality for the data distribution. None of them addresses the issue of estimating sparse $\Psi$ or $\Sigma$.

This paper is motivated by the neuroimaging data in Section 4.1. When we applied existing approaches for estimating a sparse Gaussian graphical model in $\Gamma^{-1}$ (Yuan and Lin [23]), or for estimating two sparse Gaussian graphical models in $\Psi^{-1}$ and $\Sigma^{-1}$ (Leng and Tang [13]), or for estimating a sparse covariance matrix in $\Gamma$ (Cui, Leng and Sun [8]), they all give a final estimated $\Gamma$ which is diagonal. A formal test of the null hypothesis that the covariance matrix is diagonal, however, is rejected (Chen, Zhang and Zhong [7]). On the other hand, the proposed class of estimators, collectively named sparse Kronecker-structured estimators for huge dimensional $\Psi$ and $\Sigma$ under sparsity assumptions, is found to be useful for depicting the correlation structures in $\Psi$ and $\Sigma$. See Figure 6. At the core of these estimators is to estimate non-iteratively two correlation matrices by convex optimization, one for $\Psi$ and the other for $\Sigma$. The resulting estimates are guaranteed positive definite. The technical tools used for the non-asymptotic analysis are totally different from those in Leng and Tang [13] and Zhou [26] and can be of independent interest. By "non-asymptotic analysis" we here mean that the sample size $n$ does not need to go to infinity. Apart from this, there are two major innovations in our non-asymptotic analysis. First, the non-asymptotic results cover not only the usual Gaussian distribution, but also distributions such as the exponential tail type distributions (Cai and Liu [6]) and the Bernoulli distribution, substantially enhancing the usefulness of the method. Second, our model allows the dimensionality to be arbitrary order even when the sample size is fixed, thanks to the Kronecker structure assumption that greatly reduces the number of parameters needed. For modelling covariance of random vectors, the dimensionality is allowed at most to be of sub-exponential order of the sample size (Bickel and Levina [3,4]). Methodologically, the proposed method for matrix data can be easily extended to study array data, which is straightforward operationally and theoretically, and is discussed in the paper. Our non-asymptotic analysis indicates that the proposed method gives fast rate of convergence for estimating $\Gamma$. As a result, simple estimates by soft thresholding 
usually suffice to guarantee its positive definiteness in contrast to the thresholding estimator for the covariance matrix of vector data where special care is recommended (Rothman [15], Xue, Ma and Zou [21]). This is in sharp contrast to the more difficult problem of estimation concentration matrices where loss function based approaches have to be employed (Leng and Tang [13]).

The following notations are used throughout the paper. For a square matrix $A=\left(a_{i j}\right) \in \mathbb{R}^{m \times m}$, $\operatorname{diag}(A)$ denotes a matrix consisting of the diagonal terms of $A$ as $\operatorname{diag}\left\{a_{11}, \ldots, a_{m m}\right\}$. The Frobenius norm of $A$ is denoted as $\|A\|_{F}$ and its element $\ell_{1}$ norm is denoted as $|A|_{1}$. We write $|A|_{\max }=\max _{i, j}\left|a_{i j}\right|$ as the maximum entry of $A$. The spectral norm of $A$ is $\|A\|_{2}$ denoting the largest singular value of $A$. The trace of a square matrix $A$ is denoted $\operatorname{as} \operatorname{tr}(A)$ and the Kronecker product between matrices is denoted by $\otimes$. A positive semi-definite matrix is denoted by $A \succeq 0$. The $(i, j)$ th entry of $A$ is denoted either as $a_{i j}$ or $A_{i j}$. If $A$ denotes a covariance matrix, the corresponding correlation matrix is denoted as $R^{A}$ such that $\{\operatorname{diag}(A)\}^{1 / 2} R^{A}\left\{\operatorname{diag}(A)^{1 / 2}\right\}=A$. Finally, $I_{k}$ denotes the $k \times k$ identity matrix.

The rest of the paper is organised as follows. We present the proposed Kronecker-structured estimation method in Section 2, where several variants of the approach are discussed. In Section 3, we provide the main theory, and outline the generalisation of the proposed method for estimating the covariance matrix of array data. Simulation studies and data analysis are presented in Section 4 with a short conclusion in Section 5. All the proofs are relegated to the Appendix.

\section{Kronecker-structured estimation}

We make the following assumption on the matrix variate data $X$ that includes multivariate normality as a special case.

Condition (A). The matrix variate data $X$ have the structure

$$
X=B S A^{\top},
$$

where $A, B$ are square matrices such that $A A^{\top}=\Psi, B B^{\top}=\Sigma$ and the entries of $S=\left(s_{i j}\right)$ are independent and identically distributed with mean 0 and variance 1 .

First we derive a simple sample estimate for $\Gamma=\Psi \otimes \Sigma$. Write

$$
\Psi_{n}=\frac{1}{n} \sum_{k=1}^{n} X_{k}^{\top} X_{k}, \quad \Sigma_{n}=\frac{1}{n} \sum_{k=1}^{n} X_{k} X_{k}^{\top} .
$$

Clearly, the expectations of these two matrices satisfy

$$
E\left(\Psi_{n}\right)=\operatorname{tr}(\Sigma) \Psi, \quad E\left(\Sigma_{n}\right)=\operatorname{tr}(\Psi) \Sigma,
$$

respectively, giving rise to a reasonable estimate of $\Gamma=\Psi \otimes \Sigma$ as $\Psi_{n} \otimes \Sigma_{n} /(\operatorname{tr}(\Sigma) \operatorname{tr}(\Psi))$. Noting that $\operatorname{tr}(\Gamma)=\operatorname{tr}(\Sigma) \operatorname{tr}(\Psi)$, we can estimate $\operatorname{tr}(\Sigma) \operatorname{tr}(\Psi)$ consistently by $\sum_{k=1}^{n}\left\|X_{k}\right\|_{F}^{2} / n$. Therefore, 
a simple sample estimate of $\Gamma$ admitting the Kronecker structure is

$$
\Gamma_{n}=\Psi_{n} \otimes \Sigma_{n} /\left(\frac{1}{n} \sum_{k=1}^{n}\left\|X_{k}\right\|_{F}^{2}\right) .
$$

If the sample size goes to infinity and the dimensionality of the covariance matrix is fixed, it is not difficult to see that $\Gamma_{n}$ is a consistent estimator of $\Gamma$.

Next, we discuss how to estimate $\Gamma$ when $p$ and $q$ are much larger than $n$. Define the marginal variance matrices of $\Psi_{n}$ and $\Sigma_{n}$ as

$$
\left(W_{1}^{\Psi}\right)^{2}=\operatorname{diag}\left(\Psi_{n}\right), \quad\left(W_{1}^{\Sigma}\right)^{2}=\operatorname{diag}\left(\Sigma_{n}\right),
$$

respectively. The sample row and column sample correlation matrices can be written respectively as $R_{n}^{\Psi}$ and $R_{n}^{\Sigma}$, where

$$
\Psi_{n}=W_{1}^{\Psi} R_{n}^{\Psi} W_{1}^{\Psi}, \quad \Sigma_{n}=W_{1}^{\Sigma} R_{n}^{\Sigma} W_{1}^{\Sigma}
$$

These two sample correlation matrices can be seen as estimates of the population correlation matrices $R^{\Psi}$ and $R^{\Sigma}$, respectively. The proposed Kronecker estimator replaces $R_{n}^{\Psi}$ and $R_{n}^{\Sigma}$ by their penalized estimates in

$$
\begin{aligned}
& \widehat{R}^{\Psi}=\arg \min _{R \in \mathbb{R}^{q \times q}} \frac{1}{2}\left\|R-R_{n}^{\Psi}\right\|_{F}^{2}+\lambda_{\Psi}|R|_{1}, \quad \text { s.t. } R \succeq \epsilon I_{q}, R_{j j}=1, j=1, \ldots, q, \\
& \widehat{R}^{\Sigma}=\arg \min _{R \in \mathbb{R}^{p \times p}} \frac{1}{2}\left\|R-R_{n}^{\Sigma}\right\|_{F}^{2}+\lambda_{\Sigma}|R|_{1}, \quad \text { s.t. } R \succeq \epsilon I_{p}, R_{j j}=1, j=1, \ldots, p,
\end{aligned}
$$

where $\lambda_{\Psi}$ and $\lambda_{\Sigma}$ are penalty parameters, and $\epsilon$ is an arbitrary small positive constant that guarantees positive definiteness of the estimates. Here the simplified notation $\widehat{R}^{\Psi}\left(\widehat{R}^{\Sigma}\right)$ suppresses its dependence on the sample size $n$ and the penalty parameter $\lambda_{\Psi}\left(\lambda_{\Sigma}\right)$. The optimization problem in (3) and (4) is convex and thus convex optimization techniques can be applied. In this paper, we use the efficient accelerated proximal gradient algorithm in Cui, Leng and Sun [8]. After obtaining the penalized estimates of $\Psi$ and $\Sigma$, the final Kronecker estimator assembles them as

$$
\widehat{\Psi}=W_{1}^{\Psi} \widehat{R}^{\Psi} W_{1}^{\Psi}, \quad \widehat{\Sigma}=W_{1}^{\Sigma} \widehat{R}^{\Sigma} W_{1}^{\Sigma}, \quad \widehat{\Gamma}=\widehat{\Psi} \otimes \widehat{\Sigma} /\left(\frac{1}{n} \sum_{k=1}^{n}\left\|X_{k}\right\|_{F}^{2}\right) .
$$

Motivated by the adaptive Lasso (Zou [27]), we can replace the penalty $|R|_{1}$ in (3) by $\sum_{j<k}\left|R_{j k}\right| /\left|\left(R_{n}^{\Psi}\right)_{j k}\right|$, and the penalty in (4) by $\sum_{j<k}\left|R_{j k}\right| /\left|\left(R_{n}^{\Sigma}\right)_{j k}\right|$. After the correlation matrices are obtained, we estimate $\Sigma, \Psi$ and $\Gamma$ as in (5). The estimate is denoted as $\hat{\Gamma}^{A}$ and referred to as the adaptive Kronecker estimator.

It turns out numerically that the positive definiteness constraints $R \succeq \epsilon I$ in (3) and (4) are often redundant in the sense that the resulting estimates without these constraints are positive definite. Thanks to the Kronecker product structure of $\Gamma$, the non-asymptotic analysis in the next session reveals that in estimating the $p \times p$ matrix $\Sigma$, the effective sample size becomes $n q$, and in 
estimating the $q \times q$ matrix $\Psi$, the effective sample size becomes $n p$. Thus, the curse of dimensionality is greatly alleviated. As a consequence, we can directly use thresholding as in in Bickel and Levina [4] and Rothman, Levina and Zhu [17] without the positive definite constraints, giving rise to simple and fast estimators. Although thresholding estimators for covariance of vector data are also found to be positive definite with high probability (Bickel and Levina [4], Rothman, Levina and Zhu [17]), in practice, these estimators are based on an effective sample size $n$ for estimating an $(p q) \times(p q)$ matrix in our setup, and thus are more prone to the violation of the constraints. Finally, if our estimate after thresholding is not positive definite, we then evoke the algorithm in Cui, Leng and Sun [8].

In particular, we define soft-thresholding Kronecker estimator by replacing $\widehat{R}^{\Psi}$ by $S_{\lambda_{\Psi}}\left(R_{n}^{\Psi}\right)$ and $\widehat{R}^{\Sigma}$ by $S_{\lambda_{\Sigma}}\left(R_{n}^{\Sigma}\right)$ in (5) respectively, where $S_{\lambda}$ is the soft-thresholding operator such that the $(i, j)$ th entry $(i \neq j)$ of $S_{\lambda}(A)$ is $\operatorname{sign}\left(a_{i j}\right) \cdot \max \left\{\left|a_{i j}\right|-\lambda, 0\right\}$ for some $\lambda \geq 0$, and the diagonals of $S_{\lambda}(A)$ are the same as those in $A$. These matrices can be seen as the solutions to (3) and (4) with a tuning parameter $\lambda$ but without the positive definiteness constraint $R \succeq \epsilon I$ respectively.

Similarly, define the hard-thresholding operator $H_{\lambda}(\cdot)$ such that the $(i, j)$ th entry $(i \neq j)$ of $H_{\lambda}(A)$ is $a_{i j} \cdot I\left(\left|a_{i j}\right| \geq \lambda\right)$ for some $\lambda \geq 0$ and an indicator function $I(\cdot)$. The hard-thresholding estimator $\hat{\Gamma}^{H}$ is defined by replacing $\widehat{R}^{\Psi}$ by $H_{\lambda_{\Psi}}\left(R_{n}^{\Psi}\right)$ and $\widehat{R}^{\Sigma}$ by $H_{\lambda_{\Sigma}}\left(R_{n}^{\Sigma}\right)$ in (5), respectively.

Finally, we have the covariance estimate by vectorizing $X_{k}$ as $\operatorname{vec}\left(X_{k}\right)$. Writing

$$
\widetilde{\Gamma}_{n}=\frac{1}{n} \sum_{k=1}^{n}\left\{\operatorname{vec}\left(X_{k}\right)\right\}\left\{\operatorname{vec}\left(X_{k}\right)\right\}^{\top},
$$

we can estimate a sparse correlation matrix as

$$
\widetilde{R}^{\widetilde{\Gamma}}=\arg \min _{R \in \mathbb{R}^{p q \times p q}} \frac{1}{2}\left\|R-R^{\widetilde{\Gamma}_{n}}\right\|_{F}^{2}+\lambda|R|_{1}, \quad \text { s.t. } R \succeq \epsilon I_{p q}, R_{j j}=1, j=1, \ldots, p q .
$$

We note again that the constraint $R \succeq \epsilon I$ is enforced to guarantee the positive definiteness of the resulting estimate. Without this constraint, the resulting estimate is just the soft-thresholding estimate as in Bickel and Levina [3]. However, Rothman [15] and Xue, Ma and Zou [21] observed that when the dimension $p q$ is high relative to the sample size $n$, the soft-thresholding estimator can be seriously non positive definite, giving rise to invalid covariance matrices. This phenomenon is especially true when both $p$ and $q$ are very large, a scenario that is appropriate for our study. Write $\widetilde{W}^{2}=\operatorname{diag}\left(\widetilde{\Gamma}_{n}\right)$. The vectorized estimator is formally defined as $\widetilde{\Gamma}^{V}=\widetilde{W} \widetilde{R} \tilde{\Gamma} \widetilde{W}$. More details of this approach can be found in Cui, Leng and Sun [8].

\section{Theory}

We now present non-asymptotic bounds for the proposed Kronecker estimate in terms of the spectral and Frobenius norms when $p$ and $q$ diverge to infinity. One novelty about our results is that the dimensionality $p$ and $q$ can diverge to infinity at any rate under suitable sparsity conditions, even when the sample size $n$ is fixed. This is in marked contrast to other approaches where the dimensionality is only allowed to be of sub-exponential order of the sample size, 
and the matrix is sufficiently sparse (Bickel and Levina [3,4]). Another novelty of the results is that we study distributions well beyond matrix normality, providing theoretical guarantees for studying a variety of problems much broader than those in Leng and Tang [13] and Zhou [26]. For $\Psi=\left(\psi_{i j}\right), \Sigma=\left(\sigma_{i j}\right)$, let $s_{\Psi}=\sum_{i \neq j} I\left(\psi_{i j} \neq 0\right)$ and $s_{\Sigma}=\sum_{i \neq j} I\left(\sigma_{i j} \neq 0\right)$ denote the numbers of nonzero off-diagonal parameters in $\Psi$ and $\Sigma$, respectively. The uniform upper bounds of $\psi_{i j}$ and $\sigma_{i j}$ are denoted as $\psi_{\max }$ and $\sigma_{\max }$, respectively. We impose the following conditions.

Condition (B). There exists constants $0<c_{1}<c_{2}$ such that $c_{1} \leq \lambda_{j}(\Psi) \leq c_{2}, c_{1} \leq \lambda_{i}(\Sigma) \leq c_{2}$ for $j \leq q, i \leq p$. Here $\lambda_{j}(\Psi), \lambda_{i}(\Sigma)$ are the eigenvalues of $\Psi, \Sigma$, respectively, in decreasing order.

Condition (C). Assume $p, q \rightarrow \infty, c_{4}<\frac{\log p}{\log q} \leq c_{5}$ with $c_{4}>0$.

Condition (D). We assume $E s_{i j}^{48}<\infty$.

Basically, Condition (B) states that the eigenvalues of $\Psi$ and $\Sigma$ are bounded away from zero and infinity. Hence, the diagonal elements of $\Psi$ and $\Sigma$ are also bounded from above. The model in (1) means that matrix variate $X$ is a linear transformation of some $p \times q$ variate random matrix $S$ with independent components. It generates a rich collection of $X$ from $S$ with given row and column covariance matrices $\Psi=A A^{\top}$ and $\Sigma=B B^{\top}$ such that $\operatorname{var}(\operatorname{vec}(X))=\Gamma$. In particular, if $X$ follows a matrix normal distribution, the data structure (1) is satisfied. Condition (D) is satisfied by many commonly used distributions such as the normal distribution, Bernoulli distribution and the exponential tail type distributions in Cai and Liu (2011). The moment condition can be further weakened by truncation. But we do not pursue this direction because otherwise a much lengthier proof is needed. The condition $c_{4}<\frac{\log p}{\log q} \leq c_{5}$ in (C) means that $p$ and $q$ are not necessarily in the same order. For example, we can allow $p=O\left(q^{k}\right)$ for any finite $k$.

For simplicity, we focus on the non-asymptotic bounds of the Kronecker estimate. The properties of the other estimates can be derived likewise. We first present the accuracy of the estimated correlation matrices defined in (3) and (4). Then we spell out the non-asymptotic bounds for estimating $\Psi$ and $\Sigma$ that eventually give rise to the bounds of estimating $\Gamma$.

Theorem 1. Assume that the true correlation matrix $R^{\Psi}$ and $R^{\Sigma}$ are both positive definite. Under (A)-(D) if we set the thresholding parameters as $\lambda_{\Psi}=O\left(\sqrt{\frac{\log q}{n p}}\right)$ and $\lambda_{\Sigma}=O\left(\sqrt{\frac{\log p}{n q}}\right)$, then we have

$$
\left\|\widehat{R}^{\Psi}-R^{\Psi}\right\|_{F} \leq C \sqrt{\left(s_{\Psi}+1\right) \frac{\log q}{n p}}, \text { and }\left\|\widehat{R}^{\Sigma}-R^{\Sigma}\right\|_{F} \leq C \sqrt{\left(s_{\Sigma}+1\right) \frac{\log p}{n q}}
$$

with probability $1-q^{-0.9}$ and $1-p^{-0.9}$, respectively. Here (and in what follows) $C$ is a positive constant independent of $n, p, q$ but may take different values in different places.

Note that Theorem 1 does not require the sample size $n$ to go to $\infty$. For fixed $n$, Theorem 1 continues to hold. Loosely speaking, the theorem states that in estimating $R^{\Sigma}$, the sample size becomes from $n$ to $n q$, and that it becomes $n p$ in estimating $R^{\Psi}$. Therefore, for the non-asymptotic 
results to take effect, we only need to let the effective sample sizes $n p$ and $n q$ diverge to infinity, as compared to the usual non-asymptotic arguments for which the sample size $n$ must diverge to infinity in estimating variance of vectorized data (Bickel and Levina [4]). We note that, if the vectorized estimate is used, the bound in terms of the Frobenius norm becomes $O\left(\sqrt{s_{\Gamma} \log (p q) / n}\right)$ where $s_{\Gamma}$ is the number of the nonzero off-diagonal terms of $\Gamma$ (Rothman [15]), for which a sufficient condition for the convergence is $\log (p q)=o(n)$ when $\Gamma$ is sparse. Obviously, the convergence rate of the vectorized estimate is much slower.

We have the following corollary regarding the convergence rates of the Kronecker estimates $\widehat{\Psi}$ and $\widehat{\Sigma}$ of the two covariance matrices.

Corollary 1. Assume that Conditions (A)-(D) are satisfied. We have

$$
\left\|\frac{1}{\operatorname{tr} \Sigma} \widehat{\Psi}-\Psi\right\|_{2} \leq C \sqrt{\left(s_{\Psi}+1\right) \frac{\log q}{n p}}, \quad\left\|\frac{1}{\operatorname{tr} \Sigma} \widehat{\Psi}-\Psi\right\|_{F} \leq C \sqrt{\left(s_{\Psi}+q\right) \frac{\log q}{n p}},
$$

and

$$
\left\|\frac{1}{\operatorname{tr} \Psi} \widehat{\Sigma}-\Sigma\right\|_{2} \leq C \sqrt{\left(s_{\Sigma}+1\right) \frac{\log p}{n q}}, \quad\left\|\frac{1}{\operatorname{tr} \Psi} \widehat{\Sigma}-\Sigma\right\|_{F} \leq C \sqrt{\left(s_{\Sigma}+p\right) \frac{\log p}{n q}},
$$

with probability $1-q^{-0.9}$ and $1-p^{-0.9}$, respectively.

Now, we have the following corollary regarding the rate of convergence of the Kronecker estimate $\widehat{\Gamma}$ for estimating $\Gamma$.

Corollary 2. Assume that Conditions (A)-(D) are satisfied, $\left(s_{\Psi}+q\right) \frac{\log q}{n p} \rightarrow 0$ and $\left(s_{\Sigma}+p\right) \times$ $\frac{\log p}{n q} \rightarrow 0$. We have

$$
\begin{gathered}
\|\widehat{\Gamma}-\Gamma\|_{2} \leq C\left(\sqrt{\left(s_{\Psi}+1\right) \frac{\log q}{n p}}+\sqrt{\left(s_{\Sigma}+1\right) \frac{\log p}{n q}}\right), \\
\|\widehat{\Gamma}-\Gamma\|_{F} \leq C\left(\sqrt{\left(s_{\Psi}+q\right) \frac{\log q}{n}}+\sqrt{\left(s_{\Sigma}+p\right) \frac{\log p}{n}}\right),
\end{gathered}
$$

with probability $1-p^{-0.9}-q^{-0.9}-(p q)^{-0.9}$.

As this paper is the first for studying the estimation of a sparse covariance matrix when it admits a Kronecker structure, we can only compare its rate of convergence to a few methods for estimating a sparse precision matrix. Assuming matrix normality, Leng and Tang [13] and Zhou [26] both show that their estimators have similar rates of convergence, when additional assumptions are posed on the sparsity of $\Psi^{-1}$ and $\Sigma^{-1}$. Our results improve those in Leng and Tang [13] that required $\max (p \log p / q, q \log q / p) / n \rightarrow 0$ ruling out problems with fixed $n$. In addition, the method in Leng and Tang [13] involves non-convex optimization, while Zhou [26] 
employs graphical lasso. Our method usually involves thresholding, and thus is computationally more attractive. When $p$ or $q$ is 1, the results are consistent with those in Xue, Ma and Zou [21].

We now compare the Kronecker estimate to the vectorized estimate. If we vectorize the matrix observations, the standard arguments in Xue, Ma and Zou [21] and Cui, Leng and Sun [8] can show that estimation errors satisfy

$$
\begin{aligned}
\left\|\widehat{\Gamma}^{V}-\Gamma\right\|_{2} & =O_{p}\left(\sqrt{\left(s_{\Psi} s_{\Sigma}+1\right) \frac{\log p q}{n}}\right), \\
\left\|\widehat{\Gamma}^{V}-\Gamma\right\|_{F} & =O_{p}\left(\sqrt{\left(s_{\Psi}+q\right)\left(s_{\Sigma}+p\right) \frac{\log p q}{n}}\right) .
\end{aligned}
$$

The Kronecker structure assumption on $\Gamma$ effectively increases the sample sizes to $n p$ and $n q$ for estimating $\Psi$ and $\Sigma$, respectively.

Denote the non-diagonal support of $\Psi$ as $A_{0 \Psi}=\left\{(i, j): i \neq j,(\Psi)_{i j} \neq 0\right\}$ and similarly $A_{0 \Sigma}$ for $\Sigma$. We have the following consistency results for covariance selection.

Corollary 3. Assume that Conditions (A)-(D) are satisfied.

- If $\lambda_{q}\left(R^{\Psi}\right) \gg C \sqrt{\left(s_{\Psi}+1\right) \frac{\log q}{n p}}$ and $\min _{(i, j) \in A_{0 \Psi}}\left(R^{\Psi}\right)_{i j} \gg \sqrt{\frac{\log q}{n p}}$ hold then $\left(\hat{R}^{\Psi}\right)_{i j}=0$ for $(i, j) \in A_{0 \Psi}^{C}$, and $\left(\hat{R}^{\Psi}\right)_{i j} \neq 0$ for $(i, j) \in A_{0 \Psi}$ with probability tending to one;

- If $\lambda_{p}\left(R^{\Sigma}\right) \gg C \sqrt{\left(s_{\Psi}+1\right) \frac{\log p}{n q}}$ and and $\min _{(i, j) \in A_{0 \Sigma}}\left(R^{\Sigma}\right)_{i j} \gg \sqrt{\frac{\log p}{n q}}$ then $\left(\hat{R}^{\Sigma}\right)_{i j}=0$ for $(i, j) \in A_{0 \Sigma}^{C}$, and $\left(\hat{R}^{\Sigma}\right)_{i j} \neq 0$ for $(i, j) \in A_{0 \Sigma}$ with probability tending to one.

The condition $\lambda_{q}\left(R^{\Psi}\right) \gg C \sqrt{\left(s_{\Psi}+1\right) \frac{\log q}{n p}}$ ensures that the solution to (2) without the constraint is still positive definite with probability tending to one. It is clear that the solution to (2) without this constraint becomes the soft thresholding Kronecker estimator. Therefore with probability tending to one, $\left(\hat{R}^{\Psi}\right)_{i j}=\operatorname{sign}\left(R_{n}^{\Psi}\right)_{i j}\left(\left|\left(R_{n}^{\Psi}\right)_{i j}\right|-\lambda_{\Psi}\right)_{+}$where $(b)_{+}>0$ for $b>0$, and $(b)_{+}=0$ otherwise. Corollary 3 establishes the consistency of covariance selection and is attractive from a model selection perspective.

We below discuss selection of $\lambda_{\Sigma}$ and $\lambda_{\Psi}$ via cross validation that is done by splitting the sample randomly into a training and a test set randomly $N$ times. As in Bickel and Levina [4], it suffices to prove the result when $N=1$. We consider choosing $\lambda_{\Sigma}$ and $\lambda_{\Psi}$ by a grid search on $\left\{j \sqrt{\frac{\log p}{n q}}\right\}, 0<j \leq J$ and $\left\{j \sqrt{\frac{\log p}{n q}}\right\}, 0<j \leq J_{1}$, respectively. For convenience write the observations as

$$
X_{1}, \ldots, X_{m}, X_{m+1}, \ldots, X_{m+B}
$$

with $n=m+B$, where $\left\{X_{1}, \ldots, X_{m}\right\}$ is the training set and the $\left\{X_{m+1}, \ldots, X_{m+B}\right\}$ is the test set. The two tuning parameters are chosen separately, such that for choosing $\lambda_{\Sigma}$ for example, the estimated covariance matrix of the training dataset for $\Sigma$ is the closest to the sample covariance matrix of the test set for $\Sigma$ in terms of the F norm. The selection of $\lambda_{\Psi}$ is done similarly. Denote the estimators obtained from cross validation by $\widehat{R}_{\lambda_{\Psi}}^{\Psi}$ and $\widehat{R}_{\lambda_{\Sigma}}^{\Sigma}$ respectively. Inspired by Bickel and Levina [4] we have the following theory. 
Theorem 2. Suppose that $S$ in (1) consists of i.i.d. standard normal variables. If $B=n \varepsilon_{n}$, $(\log J)^{3}=o\left(\sqrt{n q} p^{-1} \varepsilon_{n} \sqrt{\log p\left(s_{\Sigma}+p\right)}\right)$ and $J_{1}=o\left(\sqrt{n p} q^{-1} \varepsilon_{n} \sqrt{\log q\left(s_{\Psi}+q\right)}\right)$, then with probability tending to one

$$
\left\|\widehat{R}_{\lambda_{\Psi}}^{\Psi}-R^{\Psi}\right\|_{F} \leq C \sqrt{\left(s_{\Psi}+1\right) \frac{\log q}{n p}}, \quad \text { and }\left\|\widehat{R}_{\lambda_{\Sigma}}^{\Sigma}-R^{\Sigma}\right\|_{F} \leq C \sqrt{\left(s_{\Sigma}+1\right) \frac{\log p}{n q}}
$$

Corollary 4. Under the condition of Theorem 2, the following two inequalities hold with probability tending to one

$$
\left\|\widehat{\Psi}_{\lambda_{\Psi}}^{\Psi}-\Psi\right\|_{F} \leq C \sqrt{\left(s_{\Psi}+q\right) \frac{\log p}{n q}}, \quad \text { and }\left\|\frac{1}{\operatorname{tr} \Psi} \hat{\Sigma_{\lambda_{\Sigma}}}-\Sigma\right\|_{F} \leq C \sqrt{\left(s_{\Sigma}+p\right) \frac{\log p}{n q}} .
$$

In theory, a convenient choice of $\varepsilon_{n}$ is $\frac{C}{\log n}$ for some constant $C$.

\subsection{Array data}

The proposed framework can be easily extended to array-type data in a straightforward manner as we discuss below. Consider independent and identically distributed array variables $X_{k} \in$ $\mathbb{R}^{p_{1} \times \cdots \times p_{L}}, k=1, \ldots, n$. We assume that they are properly centred such that $\mathrm{E}\left(X_{k}\right)$ is a zero array and $\operatorname{var}\left(\operatorname{vec}\left(X_{k}\right)\right)=\Gamma=\Sigma_{1} \otimes \cdots \otimes \Sigma_{L}$.

As the higher-order analog of matrix rows and columns, a fiber is defined by fixing every index but one (Kolda and Bader [12]). For example, the mode- $\ell$ fibers of an array $X$ are all vectors $x_{i_{1} \cdots i_{\ell-1}: i_{\ell+1} \cdots i_{L}}$ that are obtained by fixing the values of $\left\{i_{1}, \ldots, i_{L}\right\} \backslash i_{\ell}$. The mode- $\ell$ unfolding (also known as matricization or flattening) of a tensor $X$, denoted as $X^{(\ell)}$, is an $p_{\ell} \times q_{\ell}$ matrix with $q_{\ell}=\prod_{k=1(\neq \ell)}^{L} p_{k}$ by replacing the model- $\ell$ fibers in its columns. More specifically, the $\left(i_{\ell}, j\right)$ th element is the $\left(i_{1}, \ldots, i_{L}\right)$ th element of $X$ where

$$
j=1+\prod_{k=1(\neq \ell)}^{L}\left(i_{k}-1\right) J_{k}, \quad \text { with } J_{k}=\prod_{m=1(\neq \ell)}^{k-1} p_{m}
$$

With this definition, we see that $\Sigma_{n}^{(\ell)}=\frac{1}{n} \sum_{k=1}^{n}\left\{X_{k}^{(\ell)}\right\}\left\{X_{k}^{(\ell)}\right\}^{\top}$ is an unbiased estimator of $a_{\ell} \Sigma_{\ell}$ where $a_{\ell}=\prod_{k=1(\neq \ell)}^{L} \operatorname{tr}\left(\Sigma_{k}\right)$. Thus, we have that $\bigotimes_{\ell=1}^{L} \Sigma_{n}^{(\ell)}$ is an unbiased estimate of $\left\{\prod_{\ell=1}^{L} \operatorname{tr}\left(\Sigma_{\ell}\right)\right\}^{L-1} \Gamma$. Replacing $\prod_{\ell=1}^{L} \operatorname{tr}\left(\Sigma_{\ell}\right)$ by its consistent estimate $b_{n}=\sum_{k=1}^{n}\left\|\operatorname{vec}\left(X_{k}\right)\right\|^{2} /$ $n$, we obtain a moment estimate of $\Gamma$ as

$$
\Gamma_{n}=\bigotimes_{\ell=1}^{L} \Sigma_{n}^{(\ell)} / b_{n}^{L-1}
$$

The Kronecker estimate for array data is a straightforward extension of that for matrix data. Following previous ideas, we can define various estimates similar to those in Section 2. For 
instance, we minimize (3) by replacing $R_{n}^{\Psi}$ by $R^{\Sigma_{n}^{(l)}}$ to get the Kronecker estimate of $\widehat{R}^{(\ell)}$, where $R_{n}^{\Sigma_{n}^{(l)}}$ is the correlation of $\Sigma_{n}^{(\ell)}$ and $\lambda^{(\ell)}$ is the penalty parameter. Let $\widehat{\Sigma}^{(\ell)}=W^{(\ell)} \widehat{R}^{(\ell)} W^{(\ell)}$, where $\left(W^{(\ell)}\right)^{2}=\operatorname{diag}\left(\Sigma_{n}^{(\ell)}\right)$. The final Kronecker estimate of $\Gamma$ is

$$
\widehat{\Gamma}_{\text {array }}=\bigotimes_{\ell=1}^{L} \widehat{\Sigma}^{(\ell)} / b_{n}^{L-1}
$$

To establish the asymptotic results, we can impose similar conditions as those in Section 3, under which one can show that if $\left(s_{\ell}+1\right) \frac{\log p_{\ell}}{n \prod_{k=1(\neq \ell)}^{L} p_{k}} \rightarrow 0, \ell \leq L$, we have

$$
\left\|\widehat{\Gamma}_{\text {array }}-\Gamma\right\|_{2} \leq C \sum_{\ell=1}^{L} \sqrt{\left(s_{\ell}+1\right) \frac{\log p_{\ell}}{n \prod_{k=1(\neq \ell)}^{L} p_{k}}}, \quad\left\|\widehat{\Gamma}_{\text {array }}-\Gamma\right\|_{F} \leq C \sum_{\ell=1}^{L} \sqrt{\left(s_{\ell}+p_{\ell}\right) \frac{\log p_{\ell}}{n}}
$$

with probability $1-2 \sum_{k=1}^{L} p_{k}^{-0.9}$. The detail of these results will be pursued elsewhere.

\section{Numerical study}

Extensive simulation studies are conducted to assess the finite-sample performance of the proposed estimators. In particular, we consider the following matrices as the building blocks for generating the covariance matrices $\Psi$ and $\Sigma$ throughout the simulation study. For all the simulation studies, we choose $\epsilon=10^{-6}$.

Case 1 (Banded matrix). The $(i, j)$ th entry of the matrix is $a_{i j}=\left(1-\frac{|i-j|}{5}\right)_{+}$.

Case 2 (Block diagonal matrix). Partition the indices $\{1,2, \ldots, p\}$ into $K=p / 5$ nonoverlapping subsets $I_{k}$ of equal size. Let $i_{k}$ denote the maximum index in $I_{k}$. We set

$$
a_{i j}=0.6 I_{\{i=j\}}+0.4 \sum_{i=1}^{K} I_{\left\{i \in I_{k}, j \in J_{k}\right\}}+0.4 \sum_{k=1}^{K-1}\left(I_{\left\{i=i_{k}, j \in i_{k+1}\right\}}+I_{\left\{i \in I_{k+1}, j=i_{k}\right\}}\right) .
$$

Case 3 (Random sparse matrix). Let $A=B+\delta I$ : each off-diagonal upper triangle entry in $B$ is generated independently and equals to 0.5 with probability 0.1 and 0 with probability 0.9 . The diagonals of $B$ are zero and $\delta$ is chosen such that the conditional number of $A$ is $p$.

The first two cases are similar to those in Xue, Ma and Zou [21]. Case 3, a random sparse covariance matrix, is adopted from Rothman et al. [16] and Leng and Tang [13]. The patterns of the sparsity of these three matrices can be seen from Figure 1 for $p=20$, where a random realization of the matrix in Case 3 is illustrated. We denote these three matrices as $A_{j}, j=1,2,3$.

We generate 50 data sets for each simulation setup, each data matrix taking the form $X=$ $B S A^{\top}$ where $A, B$ are square matrices such that $A A^{\top}=A_{j}, B B^{\top}=A_{k}, 3 \geq j \geq k \geq 1$ and entries of $S$ are independent $t_{10}$ random variates normalized to have variance one. We consider 


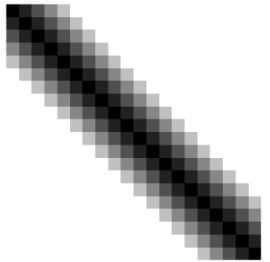

(a) Case 1

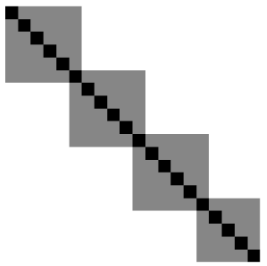

(b) Case 2

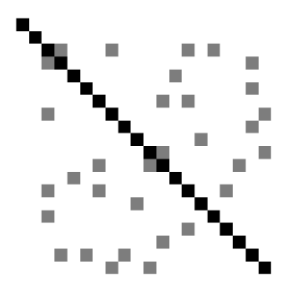

(c) Case 3

Figure 1. Heat maps of the three matrices used for simulation when $p=20$. The figure for Case 3 is random realization of the matrix generating process. Black color denotes 1 and white color denotes 0 .

a sample size $n=20$ and various dimensions as $(p, q)=(20,20),(320,320)$ or $(640,20)$. For each setup, we generate a test data with the same sample size and choose the tuning parameter that minimises the Frobenius norm of the difference between the estimate and the empirical covariance matrix of the test data.

We first examine the estimation accuracy in the Frobenius and spectral norm respectively between the truth and an estimator. The performance of various estimators of $\Gamma$ when $(p, q)=$ $(20,20)$ is presented in Figure 2. When $p q$ is large, computing the sparse vectorized estimator or a spectral norm is very slow. Thus, we only present the accuracy of estimating $\Psi$ and $\Sigma$ for $(p, q)=(320,320)$ in Figure 3 and for $(p, q)=(640,20)$ in Figure 4 respectively. Here the maximum likelihood estimator is obtained via the flip-flop algorithm in Srivastava, von Rosen and von Rosen [18] which fails to give convergent solutions if $n<\min \{p / q, q / p\}+1$ when

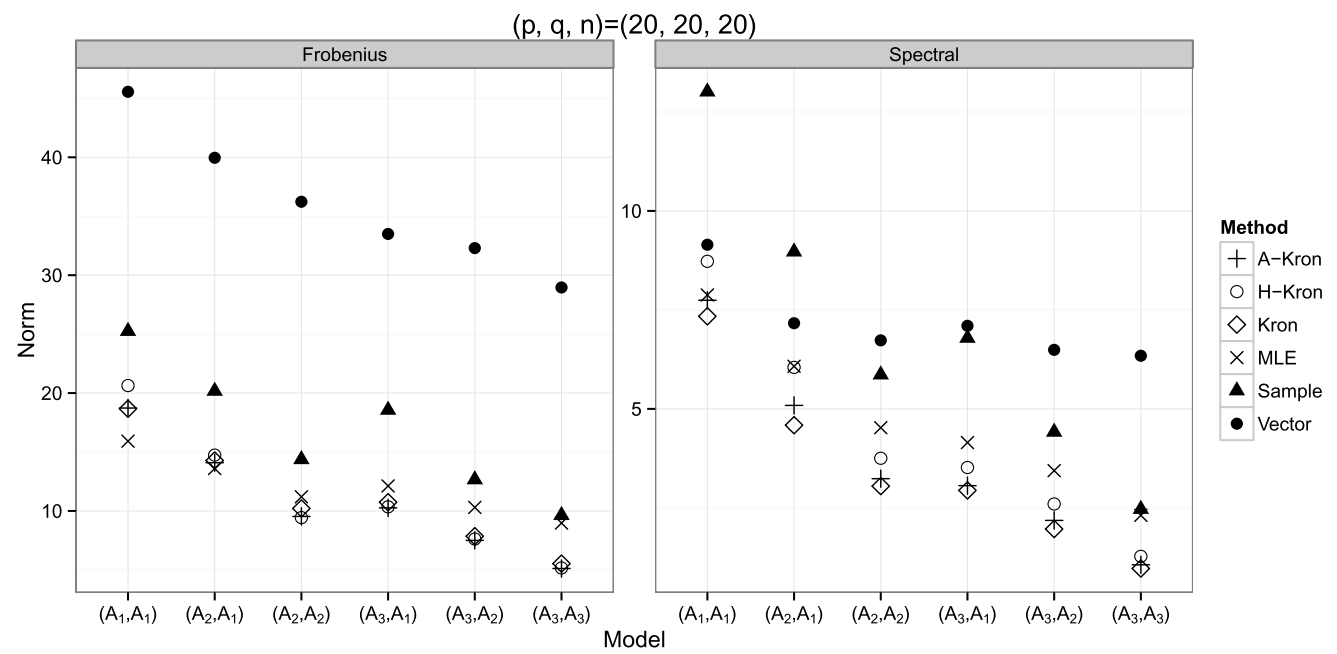

Figure 2. The accuracy when $(p, q, n)=(20,20,20)$. A-Kron: the adaptive Kronecker estimator; H-Kron: the hard-thresholding estimator; Kron: the Kronecker estimator in (5); MLE: the maximum likelihood estimator; Sample: the sample estimator in (2); Vector: the vectorized estimator. 


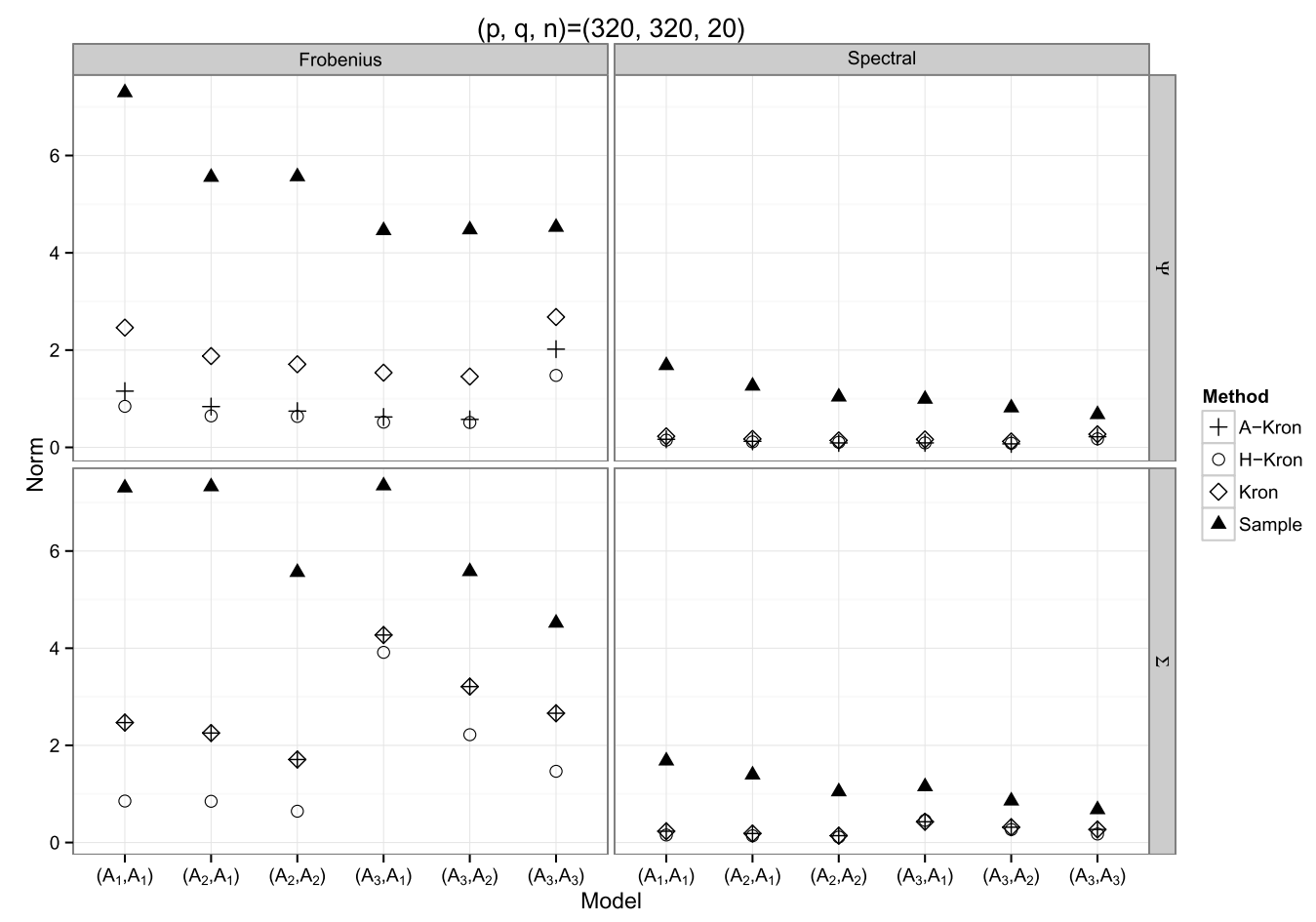

Figure 3. The accuracy when $(p, q, n)=(320,320,20)$. Short notations can be found in the caption of Figure 2.

$(p, q)=(640,20)$. This phenomenon happens because during the iteration, some of the estimated intermediate matrices are degenerate and thus cannot be used for updating. Finally, we also note that the naive sample estimator $\tilde{\Gamma}_{n}$ is outperformed by all the estimators in Figure 2 by a large magnitude (not shown) and thus is omitted for better visualization.

We can draw the following conclusions from these figures. The sample estimator and the sparse vectorized estimator are outperformed by the Kronecker estimators. The maximum likelihood estimator works well when $(p, q)=(20,20)$, but loses out when dimensionality becomes large. Among the Kronecker estimators, the hard-thresholding estimator and the adaptive Kronecker estimator perform among the best, followed by the Kronecker estimator overall.

For variable selection, we follow Leng and Tang [13] to record the true positive rate defined as $\#\left\{\hat{A}_{i j} \neq 0 \& A_{i j} \neq 0\right\} / \#\left\{A_{i j} \neq 0\right\}$ and the true negative rate defined as $\#\left\{\hat{A}_{i j}=0 \& A_{i j}=0\right\} /$ $\#\left\{A_{i j}=0\right\}$. From Table 1, we can see that the Kronecker estimators perform satisfactorily in general, especially so for the hard-thresholding and adaptive estimators.

An interesting question arises regarding how robust the method is if the assumed Kronecker structure is not true. Towards this end, we generate data by assuming

$$
\Gamma=\alpha \Psi \otimes \Sigma+(1-\alpha) I,
$$




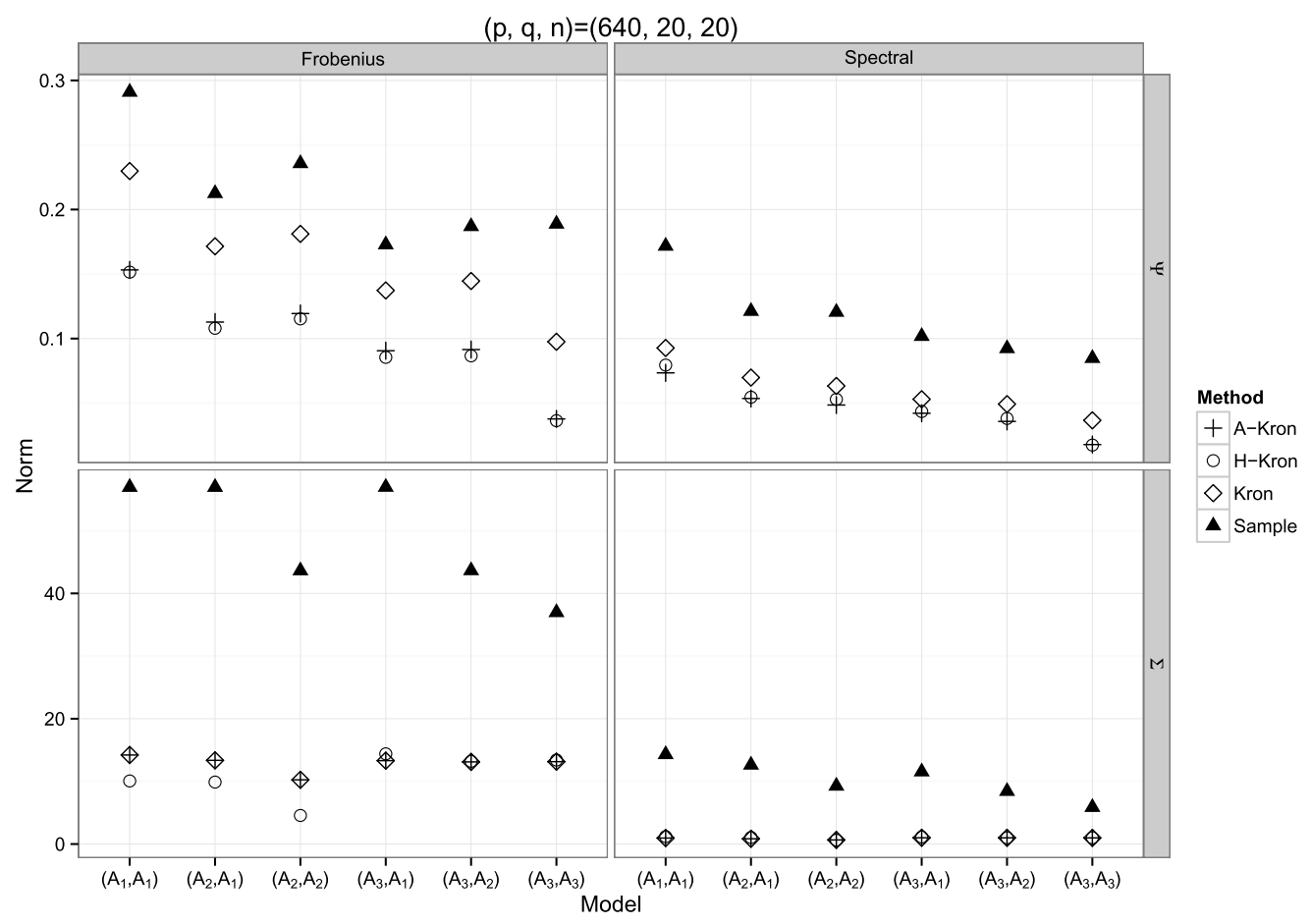

Figure 4. The accuracy when $(p, q, n)=(640,20,20)$. Short notations can be found in the caption of Figure 2.

where $\Psi$ and $\Sigma$ are specified as previously, $I$ is an $p q \times p q$ dimensional identity matrix and $\alpha \in[0,1]$ is a constant. Apparently, whenever $\alpha \in(0,1)$, the assumed Kronecker structure does not hold. We find that under this perturbation scheme, the proposed method continues to perform better than the sparse vectorized estimator, an example of which with $\alpha=0.5$ is illustrated in Figure 5. This is most likely due to the reduction of the large number of parameters, the simple structure of $\Gamma$ and the small sample size $n=20$. We have also conducted additional simulations by assuming $\Gamma=A_{i}, i=1,2,3$ which does not admit the Kronecker structure. We have observed that the proposed methods continue to outperform the sparse vectorized estimator.

\subsection{A data analysis}

As an illustration, we apply the proposed covariance matrix estimation method to analyze the Neuro Bureau ADHD-200 preprocessed data (http://www.nitrc.org/frs/?group_id=383). We examine the resting state functional magnetic resonance imaging (fMRI) data collected by Oregon Health Sciences University by focusing on the 42 typically developing children. These children served as the baseline for comparison to those diagonized with attention deficit hyperactivity disorder (ADHD). For this dataset, we examine the so-called automated anatomical labeling (AAL) 
Table 1. Model selection result in percentages. TPR, true positive rate; TNR, true negative rate; Kron, Kronecker estimator; A-Kron, adaptive Kronecker estimator; H-Kron, Kronecker estimator via hardthresholding

\begin{tabular}{|c|c|c|c|c|c|c|c|c|}
\hline \multirow[b]{2}{*}{$(\Psi, \Sigma)$} & \multirow[b]{2}{*}{ Matrix } & & \multicolumn{3}{|c|}{$(p, q, n)=(320,320,20)$} & \multicolumn{3}{|c|}{$(p, q, n)=(640,20,20)$} \\
\hline & & & Kron & A-Kron & H-Kron & Kron & A-Kron & H-Kron \\
\hline \multirow{4}{*}{$\left(A_{1}, A_{1}\right)$} & $\Psi$ & TPR & 100 & 100 & 100 & 100 & 100 & 100 \\
\hline & & TNR & 92 & 99 & 100 & 42 & 93 & 98 \\
\hline & $\Sigma$ & TPR & 100 & 100 & 100 & 92 & 88 & 78 \\
\hline & & TNR & 92 & 99 & 100 & 94 & 99 & 100 \\
\hline \multirow[t]{4}{*}{$\left(A_{2}, A_{1}\right)$} & $\Psi$ & TPR & 100 & 100 & 100 & 100 & 100 & 100 \\
\hline & & TNR & 91 & 99 & 100 & 39 & 95 & 97 \\
\hline & $\Sigma$ & TPR & 100 & 100 & 100 & 100 & 99 & 92 \\
\hline & & TNR & 92 & 99 & 100 & 95 & 99 & 100 \\
\hline \multirow[t]{4}{*}{$\left(A_{2}, A_{2}\right)$} & $\Psi$ & TPR & 100 & 100 & 100 & 100 & 100 & 100 \\
\hline & & TNR & 91 & 100 & 100 & 51 & 99 & 100 \\
\hline & $\Sigma$ & TPR & 100 & 100 & 100 & 100 & 100 & 99 \\
\hline & & TNR & 91 & 100 & 100 & 95 & 99 & 100 \\
\hline \multirow[t]{4}{*}{$\left(A_{3}, A_{1}\right)$} & $\Psi$ & TPR & 100 & 100 & 100 & 100 & 100 & 100 \\
\hline & & TNR & 85 & 100 & 100 & 45 & 96 & 99 \\
\hline & $\Sigma$ & TPR & 100 & 99 & 91 & 2 & 2 & 2 \\
\hline & & TNR & 81 & 90 & 81 & 99 & 100 & 100 \\
\hline \multirow[t]{4}{*}{$\left(A_{3}, A_{2}\right)$} & $\Psi$ & TPR & 100 & 100 & 100 & 100 & 100 & 100 \\
\hline & & TNR & 91 & 100 & 100 & 50 & 99 & 100 \\
\hline & $\Sigma$ & TPR & 100 & 100 & 99 & 7 & 2 & 2 \\
\hline & & TNR & 75 & 93 & 100 & 99 & 100 & 100 \\
\hline \multirow[t]{4}{*}{$\left(A_{3}, A_{3}\right)$} & $\Psi$ & TPR & 100 & 100 & 100 & 100 & 100 & 100 \\
\hline & & TNR & 84 & 97 & 84 & 75 & 100 & 100 \\
\hline & $\Sigma$ & TPR & 100 & 100 & 100 & 17 & 5 & 2 \\
\hline & & TNR & 84 & 97 & 100 & 97 & 99 & 100 \\
\hline
\end{tabular}

atlas with 116 regions of interest (ROI). The labels in the atlas indicate macroscopic brain structures which were obtained by fractionating the brain into functional space using nearest-neighbor interpolation (Tzourio-Mazoyer et al. [20]). In an fMRI study, brain activities are measured by detecting associated changes in blood flow through low frequency blood oxygenation level dependent (BOLD) signal in the brain. For our data, the signals of these children were recorded over 74 scans equally spaced in time. Thus, this dataset consists of $n=42$ observations, each of which can be seen as a $p \times q$ matrix with a temporal dimension $p=74$ and a spatial dimension $q=116$. 


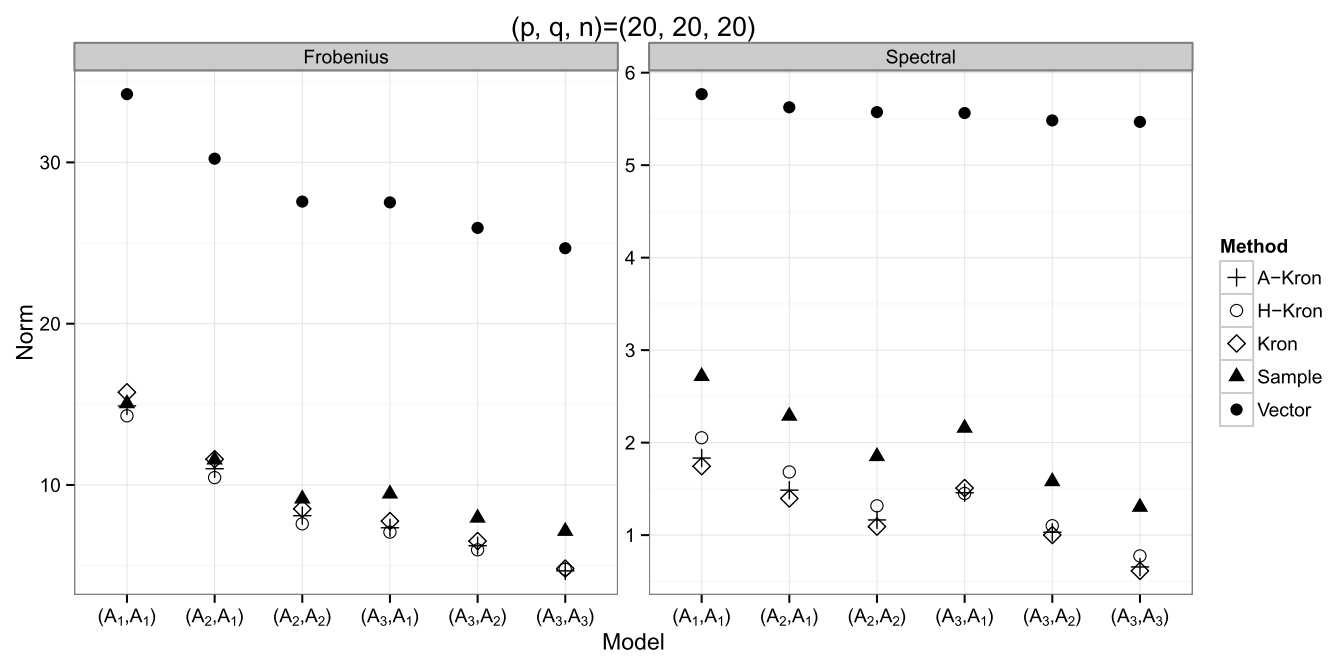

Figure 5. The accuracy when $(p, q, n)=(20,20,20)$ when the true covariate matrix does not admit the Kronecker structure. Short notations can be found in the caption of Figure 2.

To explore the covariance structure of the data, we start by vectorizing the matrices as vectors each with a dimension $p q=8584$. We fit the penalized Gaussian graphical model in Yuan and Lin [23] for estimating a sparse $\Gamma^{-1}$ and the sparse correlation matrix estimation method in Cui, Leng and Sun [8] for estimating a sparse $\Gamma$. Using 10-fold cross validation for choosing their respective tuning parameter, both method estimate $\Gamma$ as a diagonal matrix. A formal test that the $(p q) \times(p q)$ dimensional correlation matrix is an identity matrix is rejected (Chen, Zhang and Zhong [7]). We proceed to use the sparse matrix variate graphical model in Leng and Tang [13] to estimate $\Gamma$ whose inverse is represented as the Kronecker product of a sparse $\Psi^{-1}$ and $\Sigma^{-1}$. The tuning parameter is again chosen by 10 -fold cross validation and we found that both matrices are estimated as diagonal.

We then apply the Kronecker structured estimators in this paper for which 10-fold cross validation is again used to choose the tuning parameters. We first plot the sample estimators of $R^{\Psi}$ in panel (a) and $R^{\Sigma}$ in panel (d) of Figure 6. Interestingly, the correlation matrix among the scans at different times clearly exhibit a banded structure, indicating that adjacent observations along the temporal dimension are strongly correlated, while distant observations are not as correlated. This agrees with the intuition for fMRI datasets as BOLD signals at consecutive times can be related to each other. We plot the Kronecker estimator of $R^{\Psi}$ in panel (b) and $R^{\Sigma}$ in panel (e) of Figure 6. The corresponding adaptive Kronecker estimators are plotted in panels (c) and (f). We note that the adaptive Kronecker estimators are much more sparse than their non-adaptive versions. For example, the sparsity of $R^{\Sigma}$ is $74.4 \%$ when the adaptive Kronecker estimator is used, while it is $53.7 \%$ for the Kronecker estimator. Comparing the 10-fold cross validation out-of-sample errors, we further find that the errors of the Kronecker and the adaptive Kronecker estimators are much smaller than the sample estimators in panels (a) and (d) of the figure, and much better than the sample estimator when data are treated as vectors. 


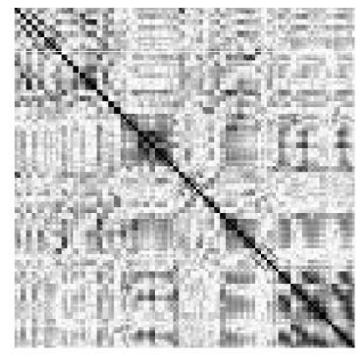

(a) Sample $R^{\Psi}$, sparsity $=0 \%$

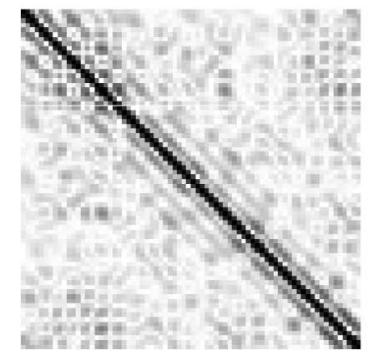

(d) Sample $R^{\Sigma}$, sparsity=0\%

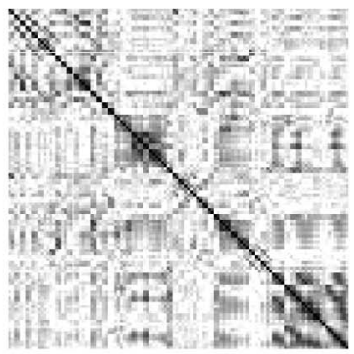

(b) Kronecker $R^{\Psi}$, sparsity $=18.6 \%$

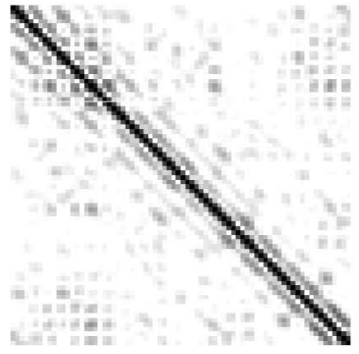

(e) Kronecker $R^{\Sigma}$, sparsity $=53.7 \%$

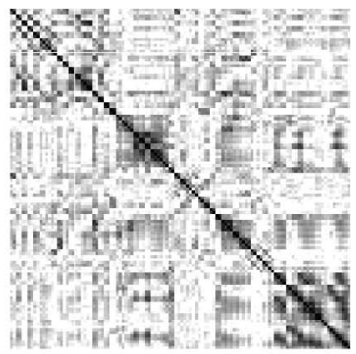

(c) Adaptive Kronecker $R^{\Psi}$, sparsity $=394 \%$

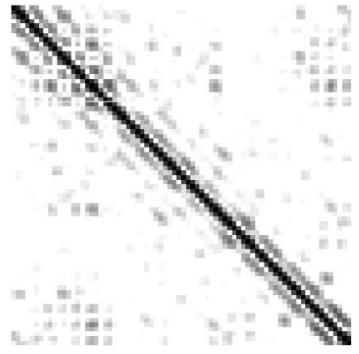

(f) Adaptive Kronecker $R^{\Sigma}$, sparsity $=74.4 \%$

Figure 6. The sample $\Psi$ and $\Sigma$ and their estimators based on the method in this paper. The sparsity is the percentage of the zeros in the corresponding matrix.

Finally, our Kronecker estimators from panels (c) and (f) confirm that a banded structure for the covariance matrix of the observed BOLD signals over the temporal domain might be appropriate.

\section{Conclusion}

We have proposed a novel sparse Kronecker structured method for estimating huge dimensional covariance matrices for matrix and array data. Our approach is simple, requiring no-iteration as opposed to iterative procedures for example, in Leng and Tang [13], easy to compute, and enjoy superior non-asymptotic results under flexible distributions as in Section 3. We impose no constraints on the dimensionality of the data compared to the sample size, as opposed to the usual practice in the literature, for example, in Bickel and Levina [4]. This highlights the significant gain in analysing high dimensional structured data by assuming a Kronecker structure on the covariance matrix. 


\section{Appendix A}

We state a roadmap of the proof to Theorem 1, consisting of two steps.

1. We first establish the following two bounds

$$
\left|\frac{1}{\operatorname{tr} \Sigma} \Psi_{n}-\Psi\right|_{\max } \leq C \sqrt{\frac{\log q}{n p}} \text { and }\left|\frac{1}{\operatorname{tr} \Psi} \Sigma_{n}-\Sigma\right|_{\max } \leq C \sqrt{\frac{\log p}{n q}}
$$

with certain probabilities. Moderate deviation for martingale is employed to establish (6) under the moment condition. To this end, a key step is to construct a martingale by appropriately rewriting the entry of the random matrix of interest. Once this is done, the next difficulty is to characterize the difference between the conditional variance and the variance of the martingale, which is accomplished by evaluating its higher moment.

2. We further derive the convergence rate of the correlation matrices by putting the correlation matrices into (3) and (4) in the main paper. Specifically, we obtain the convergence rate of $\left(\widehat{R}^{\Psi}-R^{\Psi}\right)$ and $\left(\widehat{R}^{\Sigma}-R^{\Sigma}\right)$ under the spectral norm and the Frobenius norm, respectively.

To prove (6), we first write the expression of the elements of the matrices in (6) as

$$
\begin{aligned}
& \left|\frac{\Psi_{n}}{p}-\frac{\operatorname{tr}(\Sigma) \Psi}{p}\right|_{\max }=\max _{u, v \leq q} \mid \frac{1}{n} \sum_{k=1}^{n}\left[\frac{1}{p} \sum_{i=1}^{p}\left(x_{k, i u} x_{k, i v}-\psi_{u v} \sigma_{i i}\right)\right], \\
& \left|\frac{\Sigma_{n}}{q}-\frac{\operatorname{tr}(\Psi) \Sigma}{q}\right|_{\max }=\max _{u, v \leq p} \mid \frac{1}{n} \sum_{k=1}^{n}\left[\frac{1}{q} \sum_{j=1}^{q}\left(x_{k, u j} x_{k, v j}-\psi_{j j} \sigma_{u v}\right)\right] .
\end{aligned}
$$

We then prove $(6)$ by considering the $(u, v)$ th element of the above matrices as independent sums or martingales.

For ease reference, we cite a moderate deviation result for martingales in Grama [9].

Lemma 1. Let $z_{n}$ be a martingale difference sequence with respect to the increasing $\sigma$-field $\mathfrak{F}_{n}$. Suppose that for some $\delta>0$

$$
L_{2 \delta}^{n}=E \sum_{i=1}^{n}\left|z_{i}\right|^{2+2 \delta} \rightarrow 0, \quad N_{2 \delta}^{n}=E\left|\sum_{i=1}^{n} E\left(z_{i}^{2} \mid \mathfrak{F}_{i-1}\right)-1\right|^{1+\delta} \rightarrow 0 .
$$

Suppose that $x$ is such that $1 \leq x \leq \alpha\left(L_{2 \delta}^{n}+N_{2 \delta}^{n}\right)^{-1}$ with $\alpha>0$. Then

$$
P\left(\left|\sum_{i=1}^{n} z_{i}\right| \geq r\right)=2(1-\Phi(r))\left[1+\theta C(\alpha, \delta) x^{\frac{1}{3+2 \delta}}\left(L_{2 \delta}^{n}+N_{2 \delta}^{n}\right)^{\frac{1}{3+2 \delta}}\right]
$$

where $|\theta| \leq 1, C(\alpha, \delta)$ is a constant depending only on $\alpha$ and $\delta$ and

$$
r^{2}=2 \log x-\theta_{1} 2 c(\delta) \log (1+\sqrt{2 \log x}),
$$

with $0 \leq \theta_{1} \leq 1$ and $c(\delta)=3+6 \delta$. 
Applying this lemma, we establish the following result whose proof can be found in supplementary materials.

Lemma 2. Assume that Conditions (A), (B) and (C) are satisfied. Then for some $M>0$,

$$
\begin{aligned}
& P\left(\left|\frac{\Psi_{n}}{\operatorname{tr} \Sigma}-\Psi\right|_{\max } \leq \frac{p}{\operatorname{tr} \Sigma} \sqrt{M \frac{\log q}{n p}}\right) \geq 1-q^{-0.94}, \\
& P\left(\left|\frac{\Sigma_{n}}{\operatorname{tr} \Psi}-\Sigma\right|_{\max } \leq \frac{q}{\operatorname{tr} \Psi} \sqrt{M \frac{\log p}{n q}}\right) \geq 1-p^{-0.94} .
\end{aligned}
$$

Proof of Lemma 2. Throughout the paper, we use $C$ and $C_{j}$ to denote constants which may change from line to line.

We only prove the second inequality and the first one can be proved similarly. Define

$$
Q_{q u v}=\sqrt{n q}\left[\frac{\Sigma_{n}}{q}-\frac{\operatorname{tr}(\Psi) \Sigma}{q}\right]_{u v} .
$$

By (7), write

$$
Q_{q u v}=\frac{1}{\sqrt{n q}} \sum_{k=1}^{n}\left[\sum_{j=1}^{q}\left(x_{k, u j} x_{k, v j}-\psi_{j j} \sigma_{u v}\right)\right]=\frac{1}{\sqrt{n q}} \sum_{j=1}^{q}\left[\sum_{k=1}^{n}\left(x_{k, u j} x_{k, v j}-\psi_{j j} \sigma_{u v}\right)\right] .
$$

In order to decompose $Q_{n u v}$ into a sum of some manageable terms, we introduce the following notation. Let

$$
\begin{aligned}
& A=\left(a_{i j}\right)_{q \times q}=\left(a_{1}, \ldots, a_{q}\right)^{\top}, \quad a_{i}=\left(a_{i 1}, \ldots, a_{i q}\right)^{\top}, \quad i=1, \ldots, q, \\
& B=\left(b_{i j}\right)_{p \times p}=\left(b_{1}, \ldots, b_{p}\right)^{\top}, \quad b_{i}=\left(b_{i 1}, \ldots, b_{i p}\right)^{\top}, \quad i=1, \ldots, p, \\
& S_{k}=\left(s_{k, i j}\right)_{p \times q}=\left(s_{k, 1}, \ldots, s_{k, q}\right), \quad s_{k, \ell}=\left(s_{k, 1 \ell}, \ldots, s_{k, p \ell}\right)^{\top}, \quad \ell=1, \ldots, q .
\end{aligned}
$$

Recalling (1) in the main paper and the covariance matrices $\Phi=A A^{\top}, \Sigma=B B^{\top}$, we have

$$
\begin{aligned}
\phi_{i j} & =a_{i}^{\top} a_{j}, \quad i, j \leq q, \quad \sigma_{i j}=b_{i}^{\top} b_{j}, \quad i, j \leq p, \\
x_{k, i j} & =b_{i}^{\top} S_{k} a_{j}=\sum_{\ell=1}^{q} a_{j \ell} b_{i}^{\top} s_{k, \ell} .
\end{aligned}
$$

Denote the $(i, j)$ entry of $A^{\top} A$ by $\phi_{i j}=\sum_{k=1}^{q} a_{k i} a_{k j}$.

Using (8) and (9) and the fact that $\operatorname{tr} A^{\top} A=\operatorname{tr} A A^{\top}$, we have $Q_{q u v}=\sum_{\ell=1}^{q} J_{\ell}$, where

$$
J_{\ell}=\frac{1}{\sqrt{n q}} \sum_{\alpha<\ell} \phi_{\ell \alpha}\left(J_{1 \alpha \ell}+J_{2 \alpha \ell}\right)+\frac{1}{\sqrt{n q}} \phi_{\ell \ell} J_{3 \ell}
$$


with $J_{1 \alpha \ell}=\sum_{k=1}^{n} b_{u}^{\top} s_{k, \ell} s_{k, \alpha}^{\top} b_{v}, J_{2 \alpha \ell}=\sum_{k=1}^{n} b_{u}^{\top} s_{k, \alpha} s_{k, \ell}^{\top} b_{v}$ and $J_{3 \ell}=\sum_{k=1}^{n}\left(b_{u}^{\top} s_{k, \ell} s_{k, \ell}^{\top} b_{v}-\right.$ $\left.b_{u}^{T} b_{v}\right)$. Define the $\sigma$ fields $\mathfrak{F}_{\ell}=\sigma\left(s_{k m}, k=1, \ldots, n, m=1, \ldots, \ell\right)$. Then one may verify that $E\left(J_{\ell} \mid \mathfrak{F}_{\ell-1}\right)=0$. Furthermore a direct calculation indicates that $E\left|\sum_{\ell}^{q} J_{\ell}\right|<\left(\operatorname{Var}\left(\sum_{\ell}^{q} J_{\ell}\right)\right)^{1 / 2}<$ $\infty$, as (10) below shows. Therefore, $\left\{J_{\ell}, \mathfrak{F}_{\ell}\right\}$ is a sequence of martingale differences.

We next calculate the variance of $Q_{q u v}$. One may verify that

$$
E\left(\sum_{\alpha<\ell} \phi_{\ell \alpha} J_{1 \alpha \ell}\right)^{2}=E\left(\sum_{\alpha<\ell} \phi_{\ell \alpha} J_{2 \alpha \ell}\right)^{2}=n b_{u}^{T} b_{u} b_{v}^{T} b_{v} \sum_{\alpha<\ell} \phi_{\ell \alpha}^{2}
$$

and

$$
E\left(\sum_{\alpha_{1}<\ell} \phi_{\ell \alpha_{1}} J_{1 \alpha_{1} \ell}\right)\left(\sum_{\alpha_{2}<\ell} \phi_{\ell \alpha_{2}} J_{2 \alpha_{2} \ell}\right)=n\left(b_{u}^{T} b_{v}\right)^{2} \sum_{\alpha<\ell} \phi_{\ell \alpha}^{2} .
$$

It follows that the variance of $Q_{q u v}$ is

$$
\begin{aligned}
\operatorname{Var}\left(Q_{q u v}\right)= & \sum_{\ell=1}^{q} \operatorname{Var}\left(J_{\ell}\right)=\frac{1}{n q} \sum_{\ell=1}^{q} E\left(\sum_{\alpha<\ell} \phi_{\ell \alpha}\left(J_{1 \alpha \ell}+J_{2 \alpha \ell}\right)\right)^{2}+\frac{1}{n q} \sum_{\ell=1}^{q} \phi_{\ell \ell}^{2} E\left(J_{3 \ell}\right)^{2} \\
= & \frac{b_{u}^{T} b_{u} b_{v}^{T} b_{v}+\left(b_{u}^{T} b_{v}\right)^{2}}{q} \sum_{\ell \neq \alpha}^{q} \phi_{\ell \alpha}^{2} \\
& +\frac{1}{q} \sum_{\ell=1}^{q} \phi_{\ell \ell}^{2}\left[\left(E s_{1,11}^{4}-3\right) \sum_{i=1}^{p} b_{u i}^{2} b_{v i}^{2}+b_{u}^{T} b_{u} b_{v}^{T} b_{v}+\left(b_{u}^{T} b_{v}\right)^{2}\right] .
\end{aligned}
$$

Therefore

$$
\operatorname{Var}\left(Q_{q u v}\right) \asymp \frac{\|\Phi\|_{F}^{2}}{q},
$$

where $a_{n} \asymp b_{n}$ means that there exist constants $c_{1}$ and $c_{2}$ such that $c_{1} a_{n} \leq b_{n} \leq c_{2} a_{n}$ as $n \rightarrow \infty$.

We now investigate $N_{2 \delta}^{n}$ in Lemma 1. To this end, we first evaluate the terms involved in $E\left(J_{\ell}^{2} \mid \mathfrak{F}_{\ell-1}\right)$. Note that

$$
\begin{aligned}
E\left[\left(\phi_{\ell \ell} J_{3 \ell}\right)^{2} \mid \mathfrak{F}_{\ell-1}\right] & =E\left(\phi_{\ell \ell} J_{3 \ell}\right)^{2}, \\
E\left[\left(\sum_{\alpha<\ell} \phi_{\ell \alpha} J_{1 \alpha \ell} \times \phi_{\ell \ell} J_{3 \ell}\right) \mid \mathfrak{F}_{\ell-1}\right] & =\phi_{\ell \ell} \sum_{\alpha<\ell}\left(\phi_{\ell \alpha} \sum_{k=1}^{n} s_{k, \alpha}^{T} b_{v}\right) \sum_{i=1}^{p} b_{u i}^{2} b_{v i} E s_{1,11}^{3}, \\
E\left[\left(\sum_{\alpha<\ell} \phi_{\ell \alpha} J_{2 \alpha \ell} \times \phi_{\ell \ell} J_{3 \ell}\right) \mid \mathfrak{F}_{\ell-1}\right] & =\phi_{\ell \ell} \sum_{\alpha<\ell}\left(\phi_{\ell \alpha} \sum_{k=1}^{n} b_{u}^{T} s_{k, \alpha}\right) \sum_{i=1}^{p} b_{v i}^{2} b_{u i} E s_{1,11}^{3}, \\
E\left[\left(\sum_{\alpha_{1}<\ell} \phi_{\ell \alpha_{1}} J_{1 \alpha_{1} \ell} \sum_{\alpha_{2}<\ell} \phi_{\ell \alpha_{2}} J_{2 \alpha_{2} \ell}\right) \mid \mathfrak{F}_{\ell-1}\right] & =\sum_{\alpha_{1}<\ell, \alpha_{2}<\ell}\left(\phi_{\ell \alpha_{1}} \phi_{\ell \alpha_{2}} \sum_{k=1}^{n} s_{k, \alpha_{1}}^{T} b_{v} b_{u}^{T} s_{k, \alpha_{2}}\right) b_{u}^{T} b_{v},
\end{aligned}
$$




$$
\begin{aligned}
& E\left[\left(\sum_{\alpha<\ell} \phi_{\ell \alpha} J_{1 \alpha \ell}\right)^{2} \mid \mathfrak{F}_{\ell-1}\right]=b_{u}^{\top} b_{u} \sum_{k=1}^{n}\left(\sum_{\alpha<\ell} \phi_{\ell \alpha} s_{k, \alpha}^{\top} b_{v}\right)^{2}, \\
& E\left[\left(\sum_{\alpha<\ell} \phi_{\ell \alpha} J_{2 \alpha \ell}\right)^{2} \mid \mathfrak{F}_{\ell-1}\right]=b_{v}^{\top} b_{v} \sum_{k=1}^{n}\left(\sum_{\alpha<\ell} \phi_{\ell \alpha} b_{u}^{T} s_{k, \alpha}\right)^{2} .
\end{aligned}
$$

It follows that

$$
\begin{aligned}
\sum_{\ell}^{q} E\left(J_{\ell}^{2} \mid \mathfrak{F}_{\ell-1}\right)= & \frac{1}{n q} \sum_{\ell}^{q} E\left[\left(\sum_{\alpha<\ell} \phi_{\ell \alpha}\left(J_{1 \alpha \ell}+J_{2 \alpha \ell}\right)+\phi_{\ell \ell} J_{3 \ell}\right)^{2} \mid \mathfrak{F}_{\ell-1}\right] \\
= & \frac{1}{n q} \sum_{\ell}^{q} E\left[\sum_{\alpha<\ell} \phi_{\ell \alpha}\left(J_{1 \alpha \ell}+J_{2 \alpha \ell}\right)^{2} \mid \mathfrak{F}_{\ell-1}\right]+E\left[\left(\phi_{\ell \ell} J_{3 \ell}\right)^{2} \mid \mathfrak{F}_{\ell-1}\right] \\
& +2 E\left[\sum_{\alpha<\ell} \phi_{\ell \alpha}\left(J_{1 \alpha \ell}+J_{2 \alpha \ell}\right) \phi_{\ell \ell} J_{3 \ell} \mid \mathfrak{F}_{\ell-1}\right] \\
= & \frac{1}{n q} \sum_{\ell}^{q}\left[b_{u}^{\top} b_{u} \sum_{k=1}^{n}\left(\sum_{\alpha<\ell} \phi_{\ell \alpha} s_{k, \alpha}^{\top} b_{v}\right)^{2}+b_{v}^{\top} b_{v} \sum_{k=1}^{n}\left(\sum_{\alpha<\ell} \phi_{\ell \alpha} b_{u}^{T} s_{k, \alpha}\right)^{2}\right. \\
& \left.+2 \sum_{\alpha_{1}<\ell, \alpha_{2}<\ell}\left(\phi_{\ell \alpha_{1}} \phi_{\ell \alpha_{2}} \sum_{k=1}^{n} s_{k, \alpha_{1}}^{T} b_{v} b_{u}^{T} s_{k, \alpha_{2}}\right) b_{u}^{T} b_{v}+E\left(\phi_{\ell \ell} J_{3 \ell}\right)^{2}\right] \\
& +Q_{n 8}+Q_{n 9},
\end{aligned}
$$

where

$$
Q_{n 8}=2 E s_{1,11}^{3} \frac{1}{n q} \sum_{\ell}^{q} \phi_{\ell \ell}\left[\sum_{\alpha<\ell}\left(\phi_{\ell \alpha} \sum_{k=1}^{n} s_{k, \alpha}^{\top} b_{v}\right) \sum_{i=1}^{p} b_{u i}^{2} b_{v i}\right],
$$

and

$$
Q_{n 9}=2 E s_{1,11}^{3} \frac{1}{n q} \sum_{\ell}^{q} \phi_{\ell \ell}\left[\sum_{\alpha<\ell}\left(\phi_{\ell \alpha} \sum_{k=1}^{n} b_{u}^{T} s_{k, \alpha}\right) \sum_{i=1}^{p} b_{v i}^{2} b_{u i}\right] .
$$

We conclude from (10) and (11) that

$$
\begin{aligned}
& \sum_{\ell}^{q} E\left(J_{\ell}^{2} \mid \mathfrak{F}_{\ell-1}\right)-\operatorname{Var}\left(\sum_{\ell}^{q} J_{\ell}\right) \\
& \quad=\left(Q_{n 1}+Q_{n 2}+Q_{n 3}+Q_{n 4}+Q_{n 5}+Q_{n 6}+Q_{n 7}+Q_{n 8}+Q_{n 9}\right),
\end{aligned}
$$


where

$$
\begin{aligned}
& Q_{n 1}=b_{u}^{T} b_{u}\left[\frac{1}{n q} \sum_{\ell}^{q} \sum_{k=1}^{n} \sum_{\alpha<\ell} \phi_{\ell \alpha}^{2}\left(s_{k, \alpha}^{\top} b_{v}\right)^{2}-\frac{b_{v}^{\top} b_{v}}{q} \sum_{\ell}^{q} \sum_{\alpha<\ell} \phi_{\ell \alpha}^{2}\right], \\
& Q_{n 2}=\frac{2 b_{u}^{T} b_{u}}{n q} \sum_{\ell}^{q} \sum_{k=1}^{n} \sum_{\alpha_{1}<\alpha_{2}<\ell} \phi_{\ell \alpha_{1}} \phi_{\ell \alpha_{2}} s_{k, \alpha_{1}}^{\top} b_{v} s_{k, \alpha_{2}}^{\top} b_{v}, \\
& Q_{n 3}=b_{v}^{T} b_{v}\left[\frac{1}{n q} \sum_{\ell}^{q} \sum_{k=1}^{n} \sum_{\alpha<\ell} \phi_{\ell \alpha}^{2}\left(b_{u}^{T} s_{k, \alpha}\right)^{2}-\frac{b_{u}^{\top} b_{u}}{q} \sum_{\ell}^{q} \sum_{\alpha<\ell} \phi_{\ell \alpha}^{2}\right], \\
& Q_{n 4}=\frac{2 b_{v}^{T} b_{v}}{n q} \sum_{\ell}^{q} \sum_{k=1}^{n} \sum_{\alpha_{1}<\alpha_{2}<\ell} \phi_{\ell \alpha_{1}} \phi_{\ell \alpha_{2}} b_{u}^{\top} s_{k, \alpha_{1}} b_{u}^{\top} s_{k, \alpha_{2}}, \\
& Q_{n 5}=2 b_{u}^{T} b_{v}\left[\frac{1}{n q} \sum_{\ell}^{q} \sum_{k=1}^{n} \sum_{\alpha<\ell}\left(\phi_{\ell \alpha}^{2} s_{k, \alpha}^{T} b_{v} b_{u}^{T} s_{k, \alpha}\right)-b_{u}^{T} b_{v} \frac{1}{q} \sum_{\ell}^{q} \sum_{\alpha<\ell}^{q} \phi_{\ell \alpha}^{2}\right], \\
& Q_{n 6}=2 b_{u}^{T} b_{v}\left[\frac{1}{n q} \sum_{\ell}^{q} \sum_{\alpha_{1}<\alpha_{2}<\ell}\left(\phi_{\ell \alpha_{1}} \phi_{\ell \alpha_{2}} \sum_{k=1}^{n} s_{k, \alpha_{1}}^{T} b_{v} b_{u}^{T} s_{k, \alpha_{2}}\right)\right]
\end{aligned}
$$

and

$$
Q_{n 7}=2 b_{u}^{T} b_{v}\left[\frac{1}{n q} \sum_{\ell}^{q} \sum_{\alpha_{2}<\alpha_{1}<\ell}\left(\phi_{\ell \alpha_{1}} \phi_{\ell \alpha_{2}} \sum_{k=1}^{n} s_{k, \alpha_{1}}^{T} b_{v} b_{u}^{T} s_{k, \alpha_{2}}\right)\right] .
$$

In order to offset $p^{2}$ caused by max in the inequality (17), below we evaluate the higher moments of $Q_{n j}, j=1, \ldots, 9$ in Lemma 3 below. By (10) and Lemma 3 we obtain

$$
\begin{aligned}
E\left|\frac{\sum_{\ell}^{q} E\left(J_{\ell}^{2} \mid \mathfrak{F}_{\ell-1}\right)}{\operatorname{Var}\left(\sum_{\ell}^{q} J_{\ell}\right)}-1\right|^{12} & \leq \frac{C q^{2}\left\|\Phi_{1}^{0}\left(\Phi_{1}^{0}\right)^{T}\right\|_{F}^{12}}{n^{6}\|\Phi\|_{F}^{24}}+\frac{C q^{6}}{n^{6}\|\Phi\|_{F}^{24}}+\frac{C\left\|\Phi_{1}^{0}\left(\Phi_{1}^{0}\right)^{T}\right\|_{F}^{6}}{n^{6}\|\Phi\|_{F}^{12}} \\
& \leq \frac{C}{n^{6} q^{3}},
\end{aligned}
$$

where we use Lemma 2.1 of Bhansali, Giraitis and Kokoszka [2].

We next consider $L_{2 \delta}^{n}$ with $\delta=11$ in condition (1). By Rosenthal's inequality

$$
E\left(J_{\ell}\right)^{24} \leq \frac{C}{n^{12} q^{12}} E\left(\sum_{\alpha<\ell} \phi_{\ell \alpha}\left(J_{1 \alpha \ell}+J_{2 \alpha \ell}\right)\right)^{24}+\frac{C}{n^{12} q^{12}} \phi_{\ell \ell}^{24} E J_{3 \ell}^{24} \leq \frac{C}{q^{12}}\left(\sum_{\alpha<\ell} \phi_{\ell \alpha}^{2}\right)^{12} .
$$

This, together with (10), yields that

$$
\frac{1}{\left(\operatorname{Var}\left(\sum_{\ell}^{q} J_{\ell}\right)\right)^{12}} \sum_{\ell=1}^{q} E\left(J_{\ell}\right)^{24} \leq \frac{C}{q^{11}},
$$

where we also use the fact that $\sum_{\alpha<\ell} \phi_{\ell \alpha}^{2}$ and $\phi_{\ell \alpha}$ are both bounded for any $\ell$. 
From (15) and (16), we see that $C q^{3} \leq \alpha\left(L_{2 \delta}^{n}+N_{2 \delta}^{n}\right)^{-1}$ with some appropriate $C$, independent of $q$. Therefore choose $x$ to be $C q^{3}$ in Lemma 1 . Note that $\operatorname{Var}\left(\sum_{\ell=1}^{q} J_{\ell}\right) \leq C$ by (10). It follows from Lemma 1 that

$$
\begin{aligned}
P\left(\max _{u, v \leq p}\left|\sum_{\ell=1}^{q} J_{\ell}\right| \geq M \sqrt{\log p}\right) \leq & p^{2} P\left(\left|\frac{\sum_{\ell=1}^{q} J_{\ell}}{\sqrt{\operatorname{Var}\left(\sum_{\ell=1}^{q} J_{\ell}\right)}}\right| \geq C M \sqrt{\log p}\right) \\
& \leq p^{2} P\left(\left|\frac{\sum_{\ell=1}^{q} J_{\ell}}{\sqrt{\operatorname{Var}\left(\sum_{\ell=1}^{q} J_{\ell}\right)}}\right| \geq \sqrt{2 t \log q^{3}}\right) \leq \frac{C}{p^{3 t-2}}
\end{aligned}
$$

where $M$ is chosen so that $C M \sqrt{\log p}>\sqrt{2 t \log q^{3}}$ with $2<3 t<3$. Selecting $t$ so that $3 t-2=$ 0.95 and then summarizing the above, the proof is complete.

Lemma 3. Recall the definitions of $Q_{n j}, j=1, \ldots, 9$ in (11)-(14). Then

$$
E\left(Q_{n 1}\right)^{12}+\cdots+E\left(Q_{n 9}\right)^{12} \leq \frac{C\left\|\Phi_{1}^{0}\left(\Phi_{1}^{0}\right)^{T}\right\|_{F}^{12}}{n^{6} q^{10}}+\frac{C}{n^{6} q^{6}}+\frac{C\|\Phi\|_{F}^{12}\left\|\Phi_{1}^{0}\left(\Phi_{1}^{0}\right)^{T}\right\|_{F}^{6}}{n^{6} q^{12}},
$$

where $\Phi_{1}^{0}=\left(\phi_{\ell \alpha}^{0}\right)$ stands for the matrix obtained from $A^{\top} A=\left(\phi_{\ell \alpha}\right)$ with $\phi_{\ell \alpha}^{0}=\phi_{\ell \alpha}$ if $\alpha<\ell$ and zero otherwise.

Proof of Lemma 3. Note that the terms $Q_{n 2}, Q_{n 4}, Q_{n 6}$ and $Q_{n 7}$ are similar (their upper bounds are the same up to the constants involving $\left.b_{u}^{T} b_{v}, b_{u}^{T} b_{u}, b_{v}^{T} b_{v}\right)$. Therefore we only estimate $Q_{n 2}$ below. Define

$$
u_{\alpha_{1} \alpha_{2}}=\sum_{\ell>\alpha_{2}} \phi_{\ell \alpha_{1}} \phi_{\ell \alpha_{2}}, \quad v_{\alpha_{1} \alpha_{3}}=\sum_{\alpha_{2}>\max \left(\alpha_{1}, \alpha_{3}\right)} u_{\alpha_{1} \alpha_{2}} u_{\alpha_{3} \alpha_{2}}
$$

Write

$$
Q_{n 2}=\frac{2}{n q} \sum_{k=1}^{n} \sum_{\alpha_{1}<\alpha_{2}} u_{\alpha_{1} \alpha_{2}} s_{k, \alpha_{1}}^{\top} b_{v} s_{k, \alpha_{2}}^{\top} b_{v}
$$

By Rosenthal's inequality,

$$
\begin{aligned}
E\left(Q_{n 2}\right)^{12} \leq & \frac{C}{n^{12} q^{12}}\left|\sum_{k=1}^{n} E\left(\sum_{\alpha_{2}} \sum_{\alpha_{1}<\alpha_{2}} u_{\alpha_{1} \alpha_{2}} s_{k, \alpha_{1}}^{\top} b_{v} s_{k, \alpha_{2}}^{\top} b_{v}\right)^{2}\right|^{6} \\
& +\frac{C}{n^{12} q^{12}} \sum_{k=1}^{n} E\left(\sum_{\alpha_{2}} \sum_{\alpha_{1}<\alpha_{2}} u_{\alpha_{1} \alpha_{2}} s_{k, \alpha_{1}}^{\top} b_{v} s_{k, \alpha_{2}}^{\top} b_{v}\right)^{12} \\
\leq & \frac{C}{n^{6} q^{12}}\left|\sum_{\alpha_{2}} \sum_{\alpha_{1}<\alpha_{2}} u_{\alpha_{1} \alpha_{2}}^{2}\right|^{6}+\frac{C}{n^{12} q^{12}} \sum_{k=1}^{n} E\left(\sum_{\alpha_{2}}\left(\sum_{\alpha_{1}<\alpha_{2}} u_{\alpha_{1} \alpha_{2}} s_{k, \alpha_{1}}^{\top} b_{v}\right)^{2}\right)^{6}
\end{aligned}
$$




$$
\begin{aligned}
& +\frac{C}{n^{12} q^{12}} \sum_{k=1}^{n} \sum_{\alpha_{2}} E\left(\sum_{\alpha_{1}<\alpha_{2}} u_{\alpha_{1} \alpha_{2}} s_{k, \alpha_{1}}^{\top} b_{v}\right)^{12} \\
& \leq \frac{C}{n^{6} q^{12}}\left\|\Phi_{1}^{0}\left(\Phi_{1}^{0}\right)^{T}\right\|_{F}^{12}+\frac{C}{n^{12} q^{12}} \sum_{k=1}^{n} E\left(\sum_{\alpha_{1}, \alpha_{3}} v_{\alpha_{1} \alpha_{3}} s_{k, \alpha_{1}}^{\top} b_{v} s_{k, \alpha_{3}}^{\top} b_{v}\right)^{6} \\
& +\frac{C}{n^{12} q^{12}} \sum_{k=1}^{n} \sum_{\alpha_{2}}\left(\sum_{\alpha_{1}<\alpha_{2}} E\left(u_{\alpha_{1} \alpha_{2}} s_{k, \alpha_{1}}^{\top} b_{v}\right)^{2}\right)^{6} \\
& +\frac{C}{n^{12} q^{12}} \sum_{k=1}^{n} \sum_{\alpha_{2}} \sum_{\alpha_{1}<\alpha_{2}} E\left(u_{\alpha_{1} \alpha_{2}} s_{k, \alpha_{1}}^{\top} b_{v}\right)^{12} \\
& \leq \frac{C}{n^{6} q^{12}}\left\|\Phi_{1}^{0}\left(\Phi_{1}^{0}\right)^{T}\right\|_{F}^{12}+\frac{C}{n^{12} q^{12}} \sum_{k=1}^{n} E\left(\sum_{\alpha_{3}}\left(\sum_{\alpha_{1}<\alpha_{3}} v_{\alpha_{1} \alpha_{3}} s_{k, \alpha_{1}}^{\top} b_{v}\right)^{2}\right)^{3} \\
& +\frac{C}{n^{12} q^{12}} \sum_{k=1}^{n} \sum_{\alpha_{3}} E\left(\sum_{\alpha_{1}<\alpha_{3}} v_{\alpha_{1} \alpha_{3}} s_{k, \alpha_{1}}^{\top} b_{v}\right)^{6}+\frac{C}{n^{11} q^{12}} \sum_{\alpha_{2}}\left(\sum_{\alpha_{1}<\alpha_{2}} u_{\alpha_{1} \alpha_{2}}^{2}\right)^{6} \\
& \leq \frac{C}{n^{6} q^{12}}\left\|\Phi_{1}^{0}\left(\Phi_{1}^{0}\right)^{T}\right\|_{F}^{12}+\frac{C}{n^{12} q^{10}} \sum_{k=1}^{n} \sum_{\alpha_{3}} E\left(\sum_{\alpha_{1}<\alpha_{3}} v_{\alpha_{1} \alpha_{3}} s_{k, \alpha_{1}}^{\top} b_{v}\right)^{6} \\
& +\frac{C}{n^{11} q^{12}}\left(\sum_{\alpha_{2}} \sum_{\alpha_{1}<\alpha_{2}} u_{\alpha_{1} \alpha_{2}}^{2}\right)^{6} \\
& \leq \frac{C}{n^{6} q^{12}}\left\|\Phi_{1}^{0}\left(\Phi_{1}^{0}\right)^{T}\right\|_{F}^{12}+\frac{C}{n^{11} q^{10}} \sum_{\alpha_{3}}\left(\sum_{\alpha_{1}<\alpha_{3}} v_{\alpha_{1} \alpha_{3}}^{2}\right)^{3}+\frac{C}{n^{11} q^{10}} \sum_{\alpha_{3}} \sum_{\alpha_{1}<\alpha_{3}} v_{\alpha_{1} \alpha_{3}}^{6} \\
& \leq \frac{C}{n^{6} q^{12}}\left\|\Phi_{1}^{0}\left(\Phi_{1}^{0}\right)^{T}\right\|_{F}^{12}+\frac{C}{n^{11} q^{10}}\left(\sum_{\alpha_{3}} \sum_{\alpha_{1}<\alpha_{3}} v_{\alpha_{1} \alpha_{3}}^{2}\right)^{3} \\
& \leq \frac{C}{n^{6} q^{12}}\left\|\Phi_{1}^{0}\left(\Phi_{1}^{0}\right)^{T}\right\|_{F}^{12}+\frac{C}{n^{11} q^{10}}\left(\sum_{\alpha_{3}} \sum_{\alpha_{1}<\alpha_{3}} \sum_{\alpha_{2}>\alpha_{1}} u_{\alpha_{1} \alpha_{2}}^{2} \sum_{\alpha_{2}>\alpha_{3}} u_{\alpha_{3} \alpha_{2}}^{2}\right)^{3} \\
& \leq \frac{C}{n^{6} q^{10}}\left\|\Phi_{1}^{0}\left(\Phi_{1}^{0}\right)^{T}\right\|_{F}^{12},
\end{aligned}
$$

where the third step uses the fact that

$$
\sum_{\alpha_{2}}\left(\sum_{\alpha_{1}<\alpha_{2}} u_{\alpha_{1} \alpha_{2}} s_{k, \alpha_{1}}^{\top} b_{v}\right)^{2}=\sum_{\alpha_{1}, \alpha_{3}} v_{\alpha_{1} \alpha_{3}} s_{k, \alpha_{1}}^{\top} b_{v} s_{k, \alpha_{3}}^{\top} b_{v}
$$


and the fact that

$$
\sum_{\alpha_{2}} \sum_{\alpha_{1}<\alpha_{2}} u_{\alpha_{1} \alpha_{2}}^{2}=\sum_{\ell_{1}, \ell_{2}} \sum_{\alpha_{1}<\alpha_{2}<\min \left(\ell_{1}, \ell_{2}\right)} \phi_{\ell_{1} \alpha_{1}} \phi_{\ell_{1} \alpha_{2}} \phi_{\ell_{2} \alpha_{1}} \phi_{\ell_{2} \alpha_{2}} \leq\left\|\Phi_{1}^{0}\left(\Phi_{1}^{0}\right)^{T}\right\|_{F}^{2},
$$

and the step next to the last one uses Cauchy's inequality.

Since the terms $Q_{n 1}, Q_{n 3}$ and $Q_{n 5}$ are similar (their upper bounds are the same up to the constants involving $b_{u}^{T} b_{v}, b_{u}^{T} b_{u}, b_{v}^{T} b_{v}$ ) we only consider $Q_{n 1}$ next. Let $v_{\alpha}=\sum_{\ell>\alpha} \phi_{\ell \alpha}^{2}$. Write

$$
Q_{n 1}=\frac{1}{n q} \sum_{k=1}^{n} \sum_{\alpha} v_{\alpha}\left(s_{k, \alpha}^{\top} b_{v}\right)^{2}-\frac{b_{v}^{\top} b_{v}}{q} \sum_{\alpha} v_{\alpha} .
$$

It follows that

$$
\begin{aligned}
E\left|Q_{n 1}\right|^{12} \leq & \frac{C}{n^{12} q^{12}}\left(\sum_{k=1}^{n} E\left(\sum_{\alpha} v_{\alpha}\left(s_{k, \alpha}^{\top} b_{v} b_{v}^{T} s_{k, \alpha}-b_{v}^{T} b_{v}\right)\right)^{2}\right)^{6} \\
& +\frac{C}{n^{12} q^{12}} \sum_{k=1}^{n} E\left(\sum_{\alpha} v_{\alpha}\left(s_{k, \alpha}^{\top} b_{v} b_{v}^{T} s_{k, \alpha}-b_{v}^{T} b_{v}\right)\right)^{12} \\
\leq & \frac{C}{n^{12} q^{12}}\left(\sum_{k=1}^{n} \sum_{\alpha} v_{\alpha}^{2}\right)^{6}+\frac{C}{n^{12} q^{12}} \sum_{k=1}^{n} \sum_{\alpha} v_{\alpha}^{12} \leq \frac{C}{n^{6} q^{12}}\left(\sum_{\alpha} v_{\alpha}^{2}\right)^{6} \leq \frac{C}{n^{6} q^{6}}
\end{aligned}
$$

where we use the fact that $v_{\alpha}$ is bounded.

We next consider $Q_{n 8}$ only since $Q_{n 8}$ and $Q_{n 9}$ are similar (see (11) and (12)). Note that $\left|\sum_{i=1}^{p} b_{u i}^{2} b_{v i}\right|$ is bounded. Define $u_{\alpha}=\sum_{\ell>\alpha}\left(\phi_{\ell \ell} \phi_{\ell \alpha}\right)$. Rewrite $Q_{n 8}$ as

$$
Q_{n 8}=\frac{2 E s_{1,11}^{3}}{n q} \sum_{k=1}^{n} \sum_{\alpha} s_{k, \alpha}^{\top} b_{v} u_{\alpha} \sum_{i=1}^{p} b_{u i}^{2} b_{v i}
$$

By Rosenthal's inequality

$$
\begin{aligned}
E\left|Q_{n 8}\right|^{12} & \leq \frac{C}{n^{12} q^{12}}\left|\sum_{k=1}^{n} \sum_{\alpha} b_{v}^{T} b_{v} u_{\alpha}^{2}\right|^{6}+\frac{C}{n^{12} q^{12}} \sum_{k=1}^{n} E\left|\sum_{\alpha} s_{k, \alpha}^{\top} b_{v} u_{\alpha}\right|^{12} \\
& \leq \frac{C}{n^{6} q^{12}}\left|\sum_{\alpha} u_{\alpha}^{2}\right|^{6}+\frac{C}{n^{12} q^{12}} \sum_{k=1}^{n}\left|\sum_{\alpha} v_{v}^{\top} b_{v} u_{\alpha}^{2}\right|^{6}+\frac{C}{n^{12} q^{12}} \sum_{k=1}^{n} \sum_{\alpha} E\left|s_{k, \alpha}^{\top} b_{v}\right|^{12} u_{\alpha}^{12} \\
& \leq \frac{C}{n^{6} q^{12}}\left|\sum_{\alpha} u_{\alpha}^{2}\right|^{6}+\frac{C}{n^{11} q^{12}}\left|\sum_{\alpha} u_{\alpha}^{2}\right|^{6}+\frac{C}{n^{11} q^{12}} \sum_{\alpha} u_{\alpha}^{12} \\
& \leq \frac{C}{n^{6} q^{12}}\left|\sum_{\alpha} u_{\alpha}^{2}\right|^{6} \leq \frac{C\|\Phi\|_{F}^{12}\left\|\Phi_{1}^{0}\left(\Phi_{1}^{0}\right)^{T}\right\|_{F}^{6}}{n^{6} q^{12}}
\end{aligned}
$$


because

$$
\begin{aligned}
\sum_{\alpha} u_{\alpha}^{2} & =\sum_{\ell_{1}, \ell_{2}} \sum_{\alpha<\min \left(\ell_{1}, \ell_{2}\right)} \phi_{\ell_{1} \ell_{1}} \phi_{\ell_{1} \alpha_{1}} \phi_{\ell_{2} \ell_{2}} \phi_{\ell_{2} \alpha_{2}} \\
& \leq \sum_{\ell} \phi_{\ell \ell}^{2}\left(\left.\left.\sum_{\ell_{1}, \ell_{2}}\right|_{\alpha<\min \left(\ell_{1}, \ell_{2}\right)} \phi_{\ell_{1} \alpha} \phi_{\ell_{2} \alpha}\right|^{2}\right)^{1 / 2} \leq C\|\Phi\|_{F}^{2}\left\|\Phi_{1}^{0}\left(\Phi_{1}^{0}\right)^{T}\right\|_{F} .
\end{aligned}
$$

\section{Appendix B}

The aim of this section is to prove Theorem 1, Corollary 1 and Corollary 2 . To simplify the presentation, we define the following two events,

$$
\mathcal{X}_{\Psi}=\left\{\left|\frac{1}{\operatorname{tr} \Sigma} \Psi_{n}-\Psi\right|_{\max } \leq C \sqrt{\frac{\log q}{n p}}\right\}, \quad \mathcal{X}_{\Sigma}=\left\{\left|\frac{1}{\operatorname{tr} \Psi} \Sigma_{n}-\Sigma\right|_{\max } \leq C \sqrt{\frac{\log p}{n q}}\right\}
$$

where $C$ is a constant which may have different values in different places. By Lemma 2 we have the following Lemma.

Lemma 4. Assume that Conditions (A), (B) and (C) are satisfied. We have

$$
P\left(\mathcal{X}_{\Psi}\right)=1-q^{-0.94}, \quad P\left(\mathcal{X}_{\Sigma}\right)=1-p^{-0.94}
$$

In the following, to ease the presentation, we assume that the two events $\mathcal{X}_{\Psi}$ and $\mathcal{X}_{\Sigma}$ hold.

We next derive the convergence rate regarding the correlation matrices $R_{n}^{\Psi}$ and $R_{n}^{\Sigma}$.

Lemma 5. Assume that the events $\mathcal{X}_{\Psi}$ and $\mathcal{X}_{\Sigma}$ happen. We have

$$
\left|R_{n}^{\Psi}-R^{\Psi}\right|_{\max } \leq C \sqrt{\frac{\log q}{n p}}, \quad\left|R_{n}^{\Sigma}-R^{\Sigma}\right|_{\max } \leq C \sqrt{\frac{\log p}{n q}}
$$

where $C$ is a positive constant.

Proof of Lemma 5. We only prove the first inequality while the second one can be similarly proved. Recall $R_{n}^{\Psi}=\left(W_{1}^{\Psi}\right)^{-1} \Psi_{n}\left(W_{1}^{\Psi}\right)^{-1}$ and $R^{\Psi}=\left(W^{\Psi}\right)^{-1} \Psi\left(W^{\Psi}\right)^{-1}$, where $\left(W^{\Psi}\right)^{2}=$ $\operatorname{diag}(\Psi)$. When the event $\mathcal{X}_{\Psi}$ happens, we have $\left|\frac{1}{\operatorname{tr} \Sigma}\left(W_{1}^{\Psi}\right)_{i i}^{2}-\psi_{i i}\right| \leq C \sqrt{\frac{\log q}{n p}}$ for all $i \leq q$. Then, there exist positive constants $c$ and $C$ such that

$$
c \leq\left|\left(W_{1}^{\Psi}\right)_{i i} / \sqrt{\operatorname{tr} \Sigma}\right| \leq C, \quad\left|\frac{1}{\sqrt{\operatorname{tr} \Sigma}}\left(W_{1}^{\Psi}\right)_{i i}-\sqrt{\psi_{i i}}\right| \leq C \sqrt{\frac{\log q}{n p}} .
$$


It follows that

$$
\left|\left(R_{n}^{\Psi}\right)_{i j}-\left(R^{\Psi}\right)_{i j}\right|=\left|\frac{\frac{1}{\operatorname{tr} \Sigma}\left(\Psi_{n}\right)_{i j}}{\left(W_{1}^{\Psi}\right)_{i i}\left(W_{1}^{\Psi}\right)_{j j} / \operatorname{tr} \Sigma}-\frac{\psi_{i j}}{\sqrt{\psi_{i i} \psi_{j j}}}\right| \leq C \sqrt{\frac{\log q}{n p}} .
$$

Proof of Theorem 1. Suppose that the events $\mathcal{X}_{\Psi}$ and $\mathcal{X}_{\Sigma}$ happen. Let

$$
\widehat{\Delta}=\widehat{R}^{\Psi}-R^{\Psi}=\arg \min _{\Delta} F(\Delta) \quad \text { s.t. } R^{\Psi}+\Delta \succeq \epsilon I_{q} \text { and } \Delta_{j j}=0,
$$

where $F(\Delta)=\frac{1}{2}\left\|R^{\Psi}+\Delta-R_{n}^{\Psi}\right\|_{F}^{2}+\lambda_{\Psi}\left|R^{\Psi}+\Delta\right|_{1}$.

Let $\lambda_{\Psi}=C \sqrt{\frac{\log q}{n p}}$. Then by Lemma 5, we have $\left|R_{n}^{\Psi}-R^{\Psi}\right|_{\max } \leq \lambda_{\Psi}$. Let $A_{0}$ be the matrix constructed from $R^{\Psi}$ by replacing its nonzero entries with 1 and $\Delta_{A_{0}}$ be the Hadamard product $\Delta \circ A_{0}=\left(\Delta_{i j} \cdot A_{o, i j}\right)$. Consider for any $\Delta \in\left\{\Delta: \Delta=\Delta^{T}, R^{\Psi}+\Delta \succeq \epsilon I_{q}, \Delta_{j j}=0,\|\Delta\|_{F} \geq\right.$ $\left.4\left(s_{\Psi}+1\right)^{1 / 2} \lambda_{\Psi}\right\}$. Then one can see that

$$
\begin{aligned}
F(\Delta)-F(0) & =\frac{1}{2}\left\|R^{\Psi}+\Delta-R_{n}^{\Psi}\right\|_{F}^{2}-\frac{1}{2}\left\|R^{\Psi}-R_{n}^{\Psi}\right\|_{F}^{2}+\lambda_{\Psi}\left(\left|R^{\Psi}+\Delta\right|_{1}-\left|R^{\Psi}\right|_{1}\right) \\
& =\frac{1}{2}\|\Delta\|_{F}^{2}+\left\langle\Delta, R^{\Psi}-R_{n}^{\Psi}\right\rangle+\lambda_{\Psi}\left|\Delta_{A_{0}^{c}}\right|_{1}+\lambda_{\Psi}\left(\left|\Delta_{A_{0}}+R_{A_{0}}^{\Psi}\right|_{1}-\left|R_{A_{0}}^{\Psi}\right|_{1}\right) \\
& \geq \frac{1}{2}\|\Delta\|_{F}^{2}-\lambda_{\Psi}|\Delta|_{1}+\lambda_{\Psi}\left|\Delta_{A_{0}^{c}}\right|_{1}-\lambda_{\Psi}\left|\Delta_{A_{0}}\right|_{1}=\frac{1}{2}\|\Delta\|_{F}^{2}-2 \lambda_{\Psi}\left|\Delta_{A_{0}}\right|_{1} \\
& \geq \frac{1}{2}\|\Delta\|_{F}^{2}-2 \lambda_{\Psi} \sqrt{s_{\Psi}}\|\Delta\|_{F}>0 .
\end{aligned}
$$

Here in the second inequality, we use the fact that $\Delta_{j j}=0$ and

$$
\left|\Delta_{A_{0}}\right|_{1}=\sum_{i \neq j} \Delta_{i j} \cdot A_{0, i j} \leq\left(s \Psi \sum_{i, j} \Delta_{i j}^{2}\right)^{1 / 2} \leq \sqrt{s_{\Psi}}\|\Delta\|_{F} .
$$

By the convexity of the objective function $F(\Delta)$, we immediately see that the global optimizer must satisfy

$$
\left\|\widehat{R}^{\Psi}-R^{\Psi}\right\|_{F}^{2} \leq 16\left(s_{\Psi}+1\right) \lambda_{\Psi}^{2} \leq C\left(s_{\Psi}+1\right) \frac{\log q}{n p} .
$$

Appealing to the same method, we also have

$$
\left\|\widehat{R}^{\Sigma}-R^{\Sigma}\right\|_{F}^{2} \leq C(s \Psi+1) \frac{\log p}{n q} .
$$

Hence, via Lemma 4, we have proved Theorem 1.

Proof of Corollary 1. Assume that the two events $\mathcal{X}_{\Psi}$ and $\mathcal{X}_{\Sigma}$ happen. Note that

$$
\widehat{\Psi}=\widehat{W}_{1}^{\Psi} \widehat{R}^{\Psi} \widehat{W}_{1}^{\Psi}, \quad \Psi=W^{\Psi} R^{\Psi} W^{\Psi} .
$$


We have

$$
\begin{aligned}
\frac{1}{\operatorname{tr} \Sigma} \widehat{\Psi}-\Psi= & \frac{1}{\operatorname{tr} \Sigma} \widehat{W}_{1}^{\Psi}\left(\widehat{R}^{\Psi}-R^{\Psi}\right) \widehat{W}_{1}^{\Psi} \\
& +\left(\frac{1}{\sqrt{\operatorname{tr} \Sigma}} \widehat{W}_{1}^{\Psi}-W^{\Psi}\right) R^{\Psi} \frac{1}{\sqrt{\operatorname{tr} \Sigma}} \widehat{W}_{1}^{\Psi}+W^{\Psi} R^{\Psi}\left(\frac{1}{\sqrt{\operatorname{tr} \Sigma}} \widehat{W}_{1}^{\Psi}-W^{\Psi}\right) .
\end{aligned}
$$

Consider the spectral norm of $\frac{1}{\operatorname{tr} \Sigma} \widehat{\Psi}-\Psi$ first. Since $\widehat{W}_{1}^{\Psi}, W^{\Psi}$ are diagonal matrices, we obtain

$$
\left\|\frac{1}{\sqrt{\operatorname{tr} \Sigma}} \widehat{W}_{1}^{\Psi}\right\|_{2} \leq C, \quad\left\|\frac{1}{\sqrt{\operatorname{tr} \Sigma}} \widehat{W}_{1}^{\Psi}-W^{\Psi}\right\|_{2} \leq\left|\frac{1}{\sqrt{\operatorname{tr} \Sigma}} \widehat{W}_{1}^{\Psi}-W^{\Psi}\right|_{\max } \leq C \sqrt{\frac{\log q}{n p}} .
$$

Hence, by (18), we have

$$
\begin{aligned}
& \left\|\frac{1}{\operatorname{tr} \Sigma} \widehat{\Psi}-\Psi\right\|_{2} \\
& \quad \leq\left\|\frac{1}{\sqrt{\operatorname{tr} \Sigma}} \widehat{W}_{1}^{\Psi}\right\|_{2}^{2} \cdot\left\|\widehat{R}^{\Psi}-R^{\Psi}\right\|_{F}+\left\|\frac{1}{\sqrt{\operatorname{tr} \Sigma}} \widehat{W}_{1}^{\Psi}-W^{\Psi}\right\|_{2} \cdot\left\|R^{\Psi}\right\|_{2} \cdot\left\|\frac{1}{\sqrt{\operatorname{tr} \Sigma}} \widehat{W}_{1}^{\Psi}\right\|_{2} \\
& \quad+\left\|W^{\Psi}\right\|_{2} \cdot\left\|R^{\Psi}\right\|_{2} \cdot\left\|\frac{1}{\sqrt{\operatorname{tr} \Sigma}} \widehat{W}_{1}^{\Psi}-W^{\Psi}\right\|_{2} \leq C \sqrt{(s \Psi+1) \frac{\log q}{n p}} .
\end{aligned}
$$

Consider the Frobenius norm now. Note that

$$
\left\|\frac{1}{\sqrt{\operatorname{tr} \Sigma}} \widehat{W}_{1}^{\Psi}\right\|_{F} \leq C \sqrt{q}, \quad\left\|\frac{1}{\sqrt{\operatorname{tr} \Sigma}} \widehat{W}_{1}^{\Psi}-W^{\Psi}\right\|_{F} \leq \sqrt{q}\left|\frac{1}{\sqrt{\operatorname{tr} \Sigma}} \widehat{W}_{1}^{\Psi}-W^{\Psi}\right|_{\max } \leq C \sqrt{\frac{q \log q}{n p}} .
$$

The above result, together with the formula $\|A B\|_{F} \leq\|A\|_{2} \cdot\|B\|_{F}$, implies

$$
\begin{aligned}
\left\|\frac{1}{\operatorname{tr} \Sigma} \widehat{\Psi}-\Psi\right\|_{F} \leq & \left\|\frac{1}{\sqrt{\operatorname{tr} \Sigma}} \widehat{W}_{1}^{\Psi}\right\|_{2}^{2} \cdot\left\|\widehat{R}^{\Psi}-R^{\Psi}\right\|_{F} \\
& +\left\|\frac{1}{\sqrt{\operatorname{tr} \Sigma}} \widehat{W}_{1}^{\Psi}-W^{\Psi}\right\|_{F} \cdot\left\|R^{\Psi}\right\|_{2} \cdot\left\|\frac{1}{\sqrt{\operatorname{tr} \Sigma}} \widehat{W}_{1}^{\Psi}\right\|_{2} \\
& +\left\|W^{\Psi}\right\|_{2} \cdot\left\|R^{\Psi}\right\|_{2} \cdot\left\|\frac{1}{\sqrt{\operatorname{tr} \Sigma}} \widehat{W}_{1}^{\Psi}-W^{\Psi}\right\|_{F} \\
\leq & C(\sqrt{s \Psi+1}+\sqrt{q}) \sqrt{\frac{\log q}{n p}} \leq C \sqrt{(s \Psi+q) \frac{\log q}{n p}} .
\end{aligned}
$$


Similarly, these arguments also work for the estimate $\widehat{\Sigma}$. Hence when $\mathcal{X}_{\Psi}$ and $\mathcal{X}_{\Sigma}$ happen, we have

$$
\left\|\frac{1}{\operatorname{tr} \Psi} \widehat{\Sigma}-\Sigma\right\|_{2} \leq C \sqrt{\left(s_{\Sigma}+1\right) \frac{\log p}{n q}}, \quad\left\|\frac{1}{\operatorname{tr} \Psi} \widehat{\Sigma}-\Sigma\right\|_{F} \leq C \sqrt{\left(s_{\Sigma}+p\right) \frac{\log p}{n q}}
$$

Therefore, Corollary 1 follows from Lemma 4.

Proof of Corollary 2. Define the following event

$$
\mathcal{X}_{0}=\left\{\frac{1}{n p q} \sum_{k=1}^{n}\left\|X_{k}\right\|_{F}^{2}-\left(\frac{1}{q} \operatorname{tr} \Psi\right)\left(\frac{1}{p} \operatorname{tr} \Sigma\right) \leq C \sqrt{\frac{\log (p q)}{n p q}}\right\}
$$

By a tedious proof, as in Lemma 2, we have

$$
P\left(\mathcal{X}_{0} \mid \text { under condition }(\mathrm{C})\right)=1-(p q)^{-0.95} .
$$

Suppose that the events $\mathcal{X}_{\Psi}, \mathcal{X}_{\Sigma}$, and $\mathcal{X}_{0}$ happen. Applying the formulas $\|A+B\|_{F} \leq\|A\|_{F}+$ $\|B\|_{F},\|A B\|_{F} \leq\|A\|_{F}\|B\|_{2},\|A \otimes B\|_{F}=\|A\|_{F}\|B\|_{F}$ and Corollary 1, we write

$$
\begin{aligned}
& \|\widehat{\Gamma}-\Gamma\|_{F}=\left\|\frac{\widehat{\Psi} \otimes \widehat{\Sigma}}{\left(\frac{1}{n} \sum_{k=1}^{n}\left\|X_{k}\right\|_{F}^{2}\right)}-\Psi \otimes \Sigma\right\|_{F} \\
& \leq\left\|\frac{p q \widehat{\Psi} \otimes \widehat{\Sigma}}{\left(\frac{1}{n} \sum_{k=1}^{n}\left\|X_{k}\right\|_{F}^{2}\right) \cdot(\operatorname{tr} \Psi)(\operatorname{tr} \Sigma)}\right\|_{F} \cdot\left|\frac{1}{n p q} \sum_{k=1}^{n}\left\|X_{k}\right\|_{F}^{2}-\left(\frac{1}{p} \operatorname{tr} \Sigma\right)\left(\frac{1}{q} \operatorname{tr} \Psi\right)\right| \\
& +\left\|\left(\frac{\widehat{\Psi}}{\operatorname{tr}(\Sigma)}\right) \otimes\left(\frac{\widehat{\Sigma}}{\operatorname{tr}(\Psi)}\right)-\Psi \otimes \Sigma\right\|_{F} \\
& \leq \frac{p q \cdot 2\|\Psi\|_{F} \cdot 2\|\Sigma\|_{F}}{\operatorname{tr} \Psi \operatorname{tr} \Sigma} C \sqrt{\frac{\log (p q)}{n p q}}+\left\|\left(\frac{\widehat{\Psi}}{\operatorname{tr}(\Sigma)}-\Psi\right) \otimes\left(\frac{\widehat{\Sigma}}{\operatorname{tr}(\Psi)}-\Sigma\right)\right\|_{F} \\
& +\left\|\Psi \otimes\left(\frac{\widehat{\Sigma}}{\operatorname{tr}(\Psi)}-\Sigma\right)\right\|_{F}+\left\|\left(\frac{\widehat{\Psi}}{\operatorname{tr}(\Sigma)}-\Psi\right) \otimes \Sigma\right\|_{F} \\
& \leq C\left(\sqrt{\frac{\log (p q)}{n}}+\sqrt{\left(s_{\Psi}+q\right)\left(s_{\Sigma}+p\right) \frac{\log p \log q}{n^{2} p q}}\right. \\
& +\sqrt{\left(s_{\Psi}+q\right) \frac{\log q}{n}}+\sqrt{\left.\left(s_{\Sigma}+p\right) \frac{\log p}{n}\right)} \\
& \leq C\left(\sqrt{\left(s_{\Psi}+q\right) \frac{\log q}{n}}+\sqrt{\left(s_{\Sigma}+p\right) \frac{\log p}{n}}\right),
\end{aligned}
$$


where in the last inequality, by condition (B) we use the fact that $\|\Psi\|_{F} \leq \lambda_{1}(\Psi) \sqrt{q}$ and $\|\Sigma\|_{F} \leq$ $\lambda_{1}(\Sigma) \sqrt{p}$. Note that $\|A \otimes B\|_{2} \leq\|A\|_{2} \cdot\|B\|_{2}$. From Corollary 1, we have

$$
\|\widehat{\Gamma}-\Gamma\|_{2}=O_{p}\left(\sqrt{\left(s_{\Psi}+1\right) \frac{\log q}{n p}}+\sqrt{\left(s_{\Sigma}+1\right) \frac{\log p}{n q}}\right) .
$$

Therefore, Corollary 2 follows from the above inequalities and Lemma 4.

Proof of Corollary 3. By the Weyl inequality and Theorem 1, we have

$$
\lambda_{q}\left(\hat{R}^{\Psi}\right) \geq \lambda_{q}\left(R^{\Psi}\right)-\lambda_{1}\left(\hat{R}^{\Psi}-R^{\Psi}\right)>0
$$

because $\lambda_{q}\left(R^{\Psi}\right) \gg C \sqrt{(s \Psi+1) \frac{\log q}{n p}}$ and $\lambda_{1}\left(\hat{R}^{\Psi}-R^{\Psi}\right) \leq\left\|\hat{R}^{\Psi}-R^{\Psi}\right\|_{F}$. This implies that $\hat{R}^{\Psi}$ is positive definite with probability tending to one.

Note that the above argument still holds if the constraint $R^{\Psi} \geq \varepsilon I_{q}$ is removed (one may also refer to the proof of Theorem 1). It is clear that the minimizing solution to (2) in the main paper without this constraint becomes the soft thresholding covariance estimator. Therefore with probability tending to one, $\left(\hat{R}^{\Psi}\right)_{i j}=\operatorname{sgn}\left(R_{n}^{\Psi}\right)_{i j}\left(\left|\left(R_{n}^{\Psi}\right)_{i j}\right|-\lambda_{\Psi}\right)_{+}$where $(b)_{+}>0$ for $b>0$, and $(b)_{+}=0$ otherwise. In view of the assumption $\min _{(i, j) \in A_{0 \Psi}}\left(R^{\Psi}\right)_{i j} \gg \sqrt{\frac{\log q}{n p}}, \lambda_{\Psi}=O\left(\sqrt{\frac{\log q}{n p}}\right)$ and Lemma 5, we have $\left(\hat{R}^{\Psi}\right)_{i j}=0$ for $(i, j) \in A_{0 \Psi}^{C}$ and $i \neq j$, and $\left(\hat{R}^{\Psi}\right)_{i j} \neq 0$ for $(i, j) \in A_{0 \Psi}$ and $i \neq j$ with probability tending to one. One can prove a similar result for $\hat{R}^{\Sigma}$ and details are omitted here.

\section{Appendix C: Cross validation}

We below consider $\hat{\lambda}_{\Sigma}$ only and $\hat{\lambda}_{\Psi}$ can be handled similarly where $\hat{\lambda}_{\Sigma}$ and $\hat{\lambda}_{\Psi}$ are obtained from cross validation. The proof of Theorem 2 is straightforward by following that of Theorem 1 . Indeed, one should notice that Lemmas 4 and 5 have nothing to do with $\hat{\lambda}_{\Sigma}$ (which is different from the proof in Bickel and Levina [4]). Therefore, the argument for Theorem 1 is also applicable to Theorem 2 as long as $\hat{\lambda}_{\Sigma}=O_{p}\left(\sqrt{\frac{\log p}{n p}}\right)$. Theorem 2 immediately implies Corollary 4 as in the proof of Corollary 1 .

Below we also provide an alternative proof for Corollary 4 (which is enough for the resulting estimator) since our cross validation is based on sample covariance matrices. Recall that the matrix data are generated from normal random matrices $X=B S A^{T}$, where $\{S\}=\left(s_{i j}\right)$ consists of i.i.d. standard normal random variables. Denote the spectral decomposition of $A^{T} A$ by $U \Lambda U^{T}$ with $\Lambda=\operatorname{diag}\left(\lambda_{1}, \ldots, \lambda_{q}\right)$. Define $\Sigma_{1}^{0}=\frac{1}{\operatorname{tr} \Psi} B S A^{T} A S^{T} B$. Following Lemma A.2 in Bickel and Levina [4] one can prove that for symmetric matrix $V$ with $\|V\|_{F}=1$

$$
P\left(\frac{\sqrt{q}}{\sqrt{p}}\left|\operatorname{tr} V \Sigma_{1}^{0}-\sum \gamma_{j}\right| \geq t\right) \leq K e^{-\delta t(1+o(1))},
$$


where $t \rightarrow \infty, \gamma_{1}, \ldots, \gamma_{p}$ are eigenvalues of $B^{T} V B$ and $K>0, \delta>0$. Indeed, recalling the notations above (9) by the normality of $s_{i j}$ one can see that $\operatorname{tr} V \Sigma_{1}^{0}$ has the same distribution as

$$
\frac{1}{\operatorname{tr} \Psi} \sum_{j=1}^{q} \sum_{i=1}^{p} \gamma_{i} \lambda_{j} s_{i j}^{2} .
$$

Hence, one may repeat the argument for Lemma A.2 in Bickel and Levina [4] to obtain (19). Applying (19) and repeating the arguments for Lemma A.3 in Bickel and Levina [4] we have $\rho(J) \leq \frac{C(\log J)^{2} p}{q}$, where $\rho(J)$ is the upper bound involved in condition A2 of Theorem 3 in Bickel and Levina [4].

Moreover if we change all $\Omega_{p}\left(r_{n}\right)$ in Theorem 3 of Bickel and Levina [4] to all $O_{P}\left(r_{n}\right)$, the conclusion still holds. Indeed, solving the quadratic inequality in terms of $a_{n}^{1 / 2}$ (proved similarly to Theorem 3 of Bickel and Levina [4])

$$
a_{n} \leq a_{n}^{1 / 2} o_{P}\left(r_{n}^{1 / 2}\right)+r_{n}\left(1+o_{P}(1)\right)
$$

yields $a_{n}=O_{p}\left(r_{n}\right)$, so that Theorem 3 of Bickel and Levina [4] holds for $O_{P}\left(r_{n}\right)$. Then the argument for proving Theorem 4 of Bickel and Levina [4] is also applicable here. Hence, Corollary 4 follows.

\section{Acknowledgements}

We thank Prof. Holger Dette, an associate editor and two anonymous referees for their constructive comments. Leng was partly supported by the Alan Turing Institute under the EPSRC grant EP/N510129/1. Pan was partially supported by a MOE Tier 2 grant 2014-T2-2-060 and by a MOE Tier 1 Grant RG25/14 at the Nanyang Technological University, Singapore.

\section{References}

[1] Allen, G.I. and Tibshirani, R. (2010). Transposable regularized covariance models with an application to missing data imputation. Ann. Appl. Stat. 4 764-790. MR2758420

[2] Bhansali, R.J., Giraitis, L. and Kokoszka, P.S. (2007). Convergence of quadratic forms with nonvanishing diagonal. Statist. Probab. Lett. 77 726-734. MR2356512

[3] Bickel, P.J. and Levina, E. (2008). Regularized estimation of large covariance matrices. Ann. Statist. 36 199-227. MR2387969

[4] Bickel, P.J. and Levina, E. (2008). Covariance regularization by thresholding. Ann. Statist. 36 25772604. MR2485008

[5] Bien, J. and Tibshirani, R.J. (2011). Sparse estimation of a covariance matrix. Biometrika 98 807-820. MR2860325

[6] Cai, T. and Liu, W. (2011). Adaptive thresholding for sparse covariance matrix estimation. J. Amer. Statist. Assoc. 106 672-684. MR2847949

[7] Chen, S.X., Zhang, L.-X. and Zhong, P.-S. (2010). Tests for high-dimensional covariance matrices. J. Amer. Statist. Assoc. 105 810-819. MR2724863 
[8] Cui, Y., Leng, C. and Sun, D. (2016). Sparse estimation of high-dimensional correlation matrices. Comput. Statist. Data Anal. 93 390-403. MR3406221

[9] Grama, I.G. (1997). On moderate deviations for martingales. Ann. Probab. 25 152-183. MR1428504

[10] Gupta, A.K. and Nagar, D.K. (2000). Matrix Variate Distributions. Chapman \& Hall/CRC Monographs and Surveys in Pure and Applied Mathematics 104. Boca Raton, FL: Chapman \& Hall/CRC. MR1738933

[11] Hoff, P.D. (2011). Separable covariance arrays via the Tucker product, with applications to multivariate relational data. Bayesian Anal. 6 179-196. MR2806238

[12] Kolda, T.G. and Bader, B.W. (2009). Tensor decompositions and applications. SIAM Rev. 51 455-500. MR2535056

[13] Leng, C. and Tang, C.Y. (2012). Sparse matrix graphical models. J. Amer. Statist. Assoc. $1071187-$ 1200. MR3010905

[14] Li, B., Kim, M.K. and Altman, N. (2010). On dimension folding of matrix- or array-valued statistical objects. Ann. Statist. 38 1094-1121. MR2604706

[15] Rothman, A.J. (2012). Positive definite estimators of large covariance matrices. Biometrika 99733 740. MR2966781

[16] Rothman, A.J., Bickel, P.J., Levina, E. and Zhu, J. (2008). Sparse permutation invariant covariance estimation. Electron. J. Stat. 2 494-515. MR2417391

[17] Rothman, A.J., Levina, E. and Zhu, J. (2009). Generalized thresholding of large covariance matrices. J. Amer. Statist. Assoc. 104 177-186. MR2504372

[18] Srivastava, M.S., von Rosen, T. and von Rosen, D. (2008). Models with a Kronecker product covariance structure: Estimation and testing. Math. Methods Statist. 17 357-370. MR2483463

[19] Tsiligkaridis, T. and Hero, A.O. (2013). Covariance estimation in high dimensions via Kronecker product expansions. IEEE Trans. Signal Process. 61 5347-5360.

[20] Tzourio-Mazoyer, N., Landeau, B., Papathanassiou, D., Crivello, F., Etard, O., Delcroix, N., Mazoyer, B. and Joliot, M. (2002). Automated anatomical labeling of activations in SPM using a macroscopic anatomical parcellation of the MNI MRI single-subject brain. NeuroImage 15 273-289.

[21] Xue, L., Ma, S. and Zou, H. (2012). Positive-definite $\ell_{1}$-penalized estimation of large covariance matrices. J. Amer. Statist. Assoc. 107 1480-1491. MR3036409

[22] Yin, J. and Li, H. (2012). Model selection and estimation in the matrix normal graphical model. J. Multivariate Anal. 107 119-140. MR2890437

[23] Yuan, M. and Lin, Y. (2007). Model selection and estimation in the Gaussian graphical model. Biometrika 94 19-35. MR2367824

[24] Zhou, H., Li, L. and Zhu, H. (2013). Tensor regression with applications in neuroimaging data analysis. J. Amer. Statist. Assoc. 108 540-552. MR3174640

[25] Zhou, J., Bhattacharya, A., Herring, A.H. and Dunson, D.B. (2015). Bayesian factorizations of big sparse tensors. J. Amer. Statist. Assoc. 110 1562-1576. MR3449055

[26] Zhou, S. (2014). Gemini: Graph estimation with matrix variate normal instances. Ann. Statist. 42 532-562. MR3210978

[27] Zou, H. (2006). The adaptive lasso and its oracle properties. J. Amer. Statist. Assoc. 101 1418-1429. MR2279469

Received June 2016 and revised April 2017 